\title{
Comprensión de la inclusión jerárquica de clases Estudio evolutivo
}

\section{Jesús Alonso* Francisco Gutiérrez}

\author{
Universidad Autónoma de Madrid
}

\section{INTRODUCCION}

Pese a la enorme diversidad y complejidad del universo que apreciamos, los fenómenos presentan una «estructura correlacional» que configura covariaciones naturales entre los eventos (Garner, 1974, 1978). Ciertos sucesos o propiedades tienden a aparecer juntos mientras que otros raramente mantienen alguna relación. En especial el sistema cognitivo humano está capacitado para captar estas estructuras de covariación, reduciendo la variabilidad del universo a una estructura correlativa y limitada de conceptos que compartimentan la realidad en amplias categorías de equivalencia. La extensión de tales categorías se establece como un compromiso entre la necesidad de captar las discontinuidades naturales del medio (discriminabilidad o máximo contenido informacional) y la necesidad de reducir las diferencias a proporciones cognitiva y conductualmente manejables (economía cognitiva) (Rosch y col., 1976; ver Vega, 1984, cap. 7).

Las ventajas adaptativas de un sistema categorial que obtiene el máximo de información con el mínimo de recursos cognitivos son numerosas (Oerter, 1975), y de hecho, cierto grado de habilidad para la categorización está presente en la maypría de las especies animales y es necesario para su supervivencia. Sin embargo, las capacidades conceptuales humanas son de una complejidad, originalidad y flexibilidad tales que es poco probable puedan encontrarse en otras especies (Savage-Rumbaugh y otros, 1980). Ante este hecho se hace necesario determinar en concreto qué es lo específicamente humano en la formación, aprendizaje y uso de conceptos. En este sentido, Markman y Callanan (1984) mantienen que el logro intelectual de la categorización humana no es la conceptualización en sí misma, sino la tendencia a organizar sistemáticamente los conceptos formados o aprendidos dentro de sistemas, en particular dentro de sistemas clasificatorios con relaciones de inclusión jerárquicas. De hecho, los conceptos han sido caracterizados tradicionalmente en términos de clases y clases de inclusión jerárquica (Smith y Medin, 1981). Y es que esta «dimensión vertical» que constituye la inclusión parece caracterizar el sistema conceptual humano independientemente de cuál sea el punto de vista adoptado sobre la naturaleza de los conceptos formados (ver Vega, 1984, cap. 7).

En estos sistemas de organización las categorías no son independientes sino que cada cual se relaciona con las demás de divesas formas a través de los diferentes niveles jerárquicos. Básicamente cada clase o categoría está incluida dentro de otra clase más general. Esta relación de inclusión se repite en cada nivel formándose sistemas de inclusión jerárquicos o «taxonomías» que, como señala Rosch (1978), popularmente suelen tener tres niveles, de abstracción o inclusión: las categorías básicas, las

* Dirección de los autores: Unıversidad Autónoma de Madrid, Facultad de Psicología, Departamento de Diagnóstico Psicológico y Medida. Cantoblanco. 28049 Madrid. 
supraordinadas y las subordinadas (v.g. mesa de cocina-mesa-mueble).

Numerosos autores (Vigotsky, 1962; Piaget, 1964; Bruner, 1966) han elaborado sus teorías sobre el desarrollo conceptual del niño estudiando su ejecución en diversas tareas de clasificación. Pero para estudiar el desarrollo de las habilidades de clasificación jerárquica, se hace necesario determinar previamente qué es lo que puede tomarse como evidencia de que se ba logrado este tipo de organización conceptual, lo cual, extrañamente, no se ha tenido en cuenta en la mayoría de los estudios. La aplicación al mismo objeto de dos etiquetas conceptuales de diferente nivel jerárquico no parece ser criterio suficiente, dado que el niño puede memorizarlas y utilizarlas mecánicamente sin una genuina comprensión de la organización jerárquica implicada (Smith y Medin, 1981). De acuerdo con Markman y Callanan (1984) lo que se requiere, precisamente, es evidencia de que el niño comprende las relaciones de inclusión, esto es, su carácter asimétrico y transitivo.

La asimetría y transitividad de la inclusión son un tipo de relaciones de segundo orden (relaciones entre relaciones) con ciertas implicaciones lógicas que el sujeto debe manejar apropiadamente. En concreto, la asimetría de la relación inclusiva implica que «dada una clase $A$ incluida en otra $B$, todos los miembros de A son miembros de $B$, pero no a la inversa: todos los miembros de $B$ no lo son de $A$, sólo algunos». Si esta asimetría de pertenencia se comprende, se debería juzgar correctamente, por ejemplo que aunque todas las rosas son flores sólo algunas flores son rosas. Asimismo, la pertenencia asimétrica implica asimetria en cuanto a la asignación de propiedades, dado que «si una clase $A$ está incluida en otra $B$, los miembros de A poseen todas las propiedades que definen $B$, pero no a la inversa: los miembros de $\mathrm{B}$ no necesariamente poseen todas las propiedades que definen A». Además la asimetría supone una extensión diferencial jerárquica de las clases, de modo que «cuanto mayor es su nivel jerárquico mayor es el número de sus miembros». Por otro lado, la transitividad de la inclusión supone que «si una clase $A$ está incluida en otra $B$, a su vez incluida en otra $\mathrm{C}$, entonces la clase $\mathrm{A}$ está incluida en la cla- se $C »$. Así, un sujeto que comprende esta relación transitiva, si se le indica que todos los « $A$ » son perros, debería ser capaz de decidir que todos los «A» son animales. Además, esta relación transitiva de pertenencia es a su vez asimétrica: «si $A$ está incluida en B y B está incluida en $C$, A está incluida en $C$; pero si $A$ está incluida en $C$ y $B$ está incluida en $C A$ no necesariamente está incluida en $\mathrm{B}$ »; en consecuencia debería aceptarse que, siendo «A» un animal no necesariamente tiene que ser un perro.

Si bien la literatura sobre el desarrollo de la organización conceptual del niño es extensa y variada, muy pocos estudios examinan directamente la comprensión de la asimetría y transitividad de la inclusión, y, por tanto, es escasa la evidencia que se ofrece sobre la apreciación de la inclusión por parte del niño y cabe dudar de la exactitud de las evoluciones encontradas. Mientras que con el problema piagetiano clásico de inclusión de clases y algunos estudios directos de las clasificaciones de los niños, se encuentra un desarrollo por etapas que sitúan el logro de la clasificación jerárquica a los 7 u 8 años (Inhelder y Piaget, 1964; Vigotsky, 1962; Bruner y col., 1966), otras investigaciones indirectas de la clasificación infantil (v. g. estudios de habituación a ciertos conceptos, análisis de la manipulación espontánea de objetos o estudios sobre organización de materiales en la memoria) hallan categorizaciones taxonómicas incluso en los niños más pequeños (ver revisión de Markman y Callanan, 1984, Alonso y Gutiérrez, 1986). Evidentemente, la dificultad de las tareas y el procedimiento de evaluación em. pleados en la medida de la habilidad del niño, determinan su ejecución. Así, en algunas tareas (v.g. los problemas piagetianos clásicos de inclusión de clases un sujeto puede fracasar, no porque no comprenda la relación de inclusión, sino porque existen requerimientos adicionales que pueden determinar el fracaso; en otras (v.g. los estudios de «habituación» en niños muy pequeños), el niño puede exhibir una ejecución adecuada sin una verdadera comprensión de los principios que rigen la clasificación jerárquica.

A la vista de los hechos precedentes hemos realizado un nuevo estudio evolutivo que pretende precisar el desarrollo de la in- 
clusión jerárquica a partir del criterio de evaluación propuesto por Markman (1984): la apreciación de las relaciones de asimetría y transitividad entre clases. Para ello, tras una extensa revisión y análisis de las tareas y procedimientos urilizados en los estudios previos (ver Alonso y Gutiérrez, 1986) hemos seleccionado aquellos que según el criterio adoptado, permiten alguna estimación aceptable de la comprensión de las implicaciones lógicas de la inclusión; en concreto, el problema piagetiano clásico de inclusión de clases, problemas de inferencia transitiva y cuestiones sobre aplicación asimétrica de los cuantificadores «todos» y «algunos». Unicamente estos tres tipos de tareas han mostrado su utilidad como procedimientos válidos de evaluación. Hemos rechazado las tareas de clasificación explícita por poseer numerosos requerimientos adicionales (demandas espaciales de la tarea, el gran número y complejidad de los objetos a agrupar, el nivel generalmente supraordinado de las categorías - por otra parte, poco familiares-y la propia preferencia del niño por opciones temáticas frente a la clasificación basada en un criterio), que pueden impedir u ocultar la habilidad para la categorización jerárquica; y considerando, además, que el niño en este tipo de tareas puede, por el contrario, llegar a organizar los objetos en categorías taxonómicas sin una verdadera comprensión del carácter asimétrico y transitivo de la inclusión. Por razones semejantes tampoco hemos considerado los procedimientos indirectos de evaluación como el paradigma de habituación, la manipulación espontánea y las tareas de memoria.

Como veremos, la lógica involucrada en cada tipo de tarea seleccionada es diferente y recogen, en conjunto, todas las implicaciones que se derivan de la asimetría y transitividad de la inclusión: el problema clásico piagetiano incide especialmente en la extensión diferencial de las clases jerárquicas, los problemas inferenciales manejan la transitividad y asimetría de la transitividad y la aplicación de cuantificadores exige la comprensión de la asimetría simple de pertenencia. Por consiguiente, la comparación de ejecuciones en niños de las diversas edades, nos ha permitido determinar el orden y momento de adquisición de cada compo- nente lógico, aunque en principio cada tarea ha definido un experimento diferente. A fin de abarcar todas y cada una de las consecuencias lógicas de la inclusión, cada experimento se ha concretado en el diseño de una prueba objetiva de lápiz y papel (ver anexo), elaborada con distintos tipos de cuestiones relativas a cada modalidad de problema. Por otro lado, y a partir de diversas versiones de las mismas cuestiones, hemos establecido variaciones sistemáticas respecto de algunos factores importantes que, como se ha demostrado en los estudios previos, pueden suponer dificultades de procesamiento adicionales a la lógica de la tarea (ver Winer, 1980; Alonso y Gutiérrez, 1986), con lo cual hemos podido valorar también su influencia diferencial en las distintas edades. En concreto, se han considerado tres variables principales: el tipo de contenido o estructura jerárquica en sús condiciones más relevantes (el contenido de «clase» frente al de «colección»); el tipo de presentación del problema (simplemente verbal frente a la que añade elementos perceptivos) y el tipo de categorías de referencia (categorías familiares frente a categorías nuevas) ${ }^{!}$.

Al elegir estas variables hemos pretendido recoger una selección que de algún modo fuese representativa del conjunto de influencias que los distintos estudios han puesto de manifiesto. Pero a diferencia de estos trabajos previos en los que se han analizado los factores por separado (Winer, 1980), hemos considerado la posibilidad de interacción entre los mismos, lo cual nos ha permitido una evaluación más precisa de sus efectos. Así, en el presente trabajo no sólo se describe el curso del desarrollo de la lógica de la inclusión de clases como tal y en sus distintos componentes sino que además se ofrecen importantes datos sobre posibles factores responsables de este desarrollo.

\section{Muestra}

Dado el carácter evolutivo de nuestro estudio, se eligió una muestra de 180 escolares, con 20 sujetos en cada grado, desde preescolar (4-5 años) a $8^{\circ}(13-14)$. Se ha controlado la variable sexo incluyendo en cada uno de los cursos 10 niños y 10 niñas, 
Todos los sujetos eran alumnos del colegio «Antonio Machado» de Alcobendas (Madrid) y se seleccionaron al azar dentro de cada curso y en dos aulas distintas, la mitad en cada una de ellas. A partir de las indicaciones de los profesores se eliminaron de la muestra aquellos sujetos que por una $u$ otra razón (timidez, retraso extremo, "gamberrismo", etc.), cabía esperar no tomasen la prueba con la actitud y dedicación convenientes.

\section{Material y procedimiento general}

Como ya se ha indicado, la naturaleza eminentemente lógica de las tareas propuestas nos ha permitido su presentación como una prueba objetiva de lápiz y papel (ver Anexo I). En total, el test desarrollado consta de 60 items distribuidos de la siguiente forma: 40 ítems relativos a la extensión diferencial entre clases (problemas de tipo piagetiano); 12 problemas de inferencia transitiva y 8 con cuantificadores (asimetría de pertenencia). Estos tres bloques definen los tres experimentos llevados a cabo. Para cada cuestión se ofrecen dos o tres alternativas de respuesta, según los casos, de las cuales, sólo una es correcta. Así, todas las cuestiones han podido puntuarse como 1 o 0 según se diese éxito o fracaso. Las respuestas se anotaban en una hoja aparte construida a tal efecto (ver Anexo II), que reproducía las alternativas correspodientes a cada cuestión.

Todas las tareas en todas las condiciones se pasaron a todos los sujetos. Su presentación en forma de test permitió en algunos niveles el examen por grupos. Concretamente los cursos de $8^{\circ}, 7^{\circ}$ y $6^{\circ}$ se examinaron en grupos de $10^{\circ}$ y los de $5^{\circ} 4^{\circ}$ y $3^{\circ}$ en grupos de 3 . Antes del ejercicio, los sujetos recibieron las instrucciones que se detallan en el Anexo III. La cuantía de los grupos tenía por objeto lograr un adecuado control de la resolución de la prueba y poder prestar a cada individuo las atenciones oportunas en el curso de su ejecución - teniendo en cuenta que en estos casos, sólo intervino un examinador. En los grados inferiores, preescolar, $1^{\alpha}$ y $2^{o}$ se pasó la prueba individualmente, siendo el examinador quien leía las cuestiones y anotaba las res- puestas (dadas las dificultades de comprensión lectora a estos niveles), aunque en todos los casos el sujeto tenía a la vista un ejemplar de la prueba para proporcinar los elementos perceptivos correspondientes. En estos grados no se dieron instrucciones previas, pero se les orientó convenientemente durante todo el ejercicio. Además, para evitar los efectos de la fatiga y dispersión de la atención se les administró el examen en dos partes y en días consecutivos. En estos grupos fueron dos los examinadores.

\section{EXPERIMENTO 1: CUESTIONES DE TIPO PIAGETIANO; EVOLUCION DE LA COMPRENSION DE LA EXTENSION DIFERENCIAL DE CLASES}

\section{Introducción}

Según la teoría de Piaget sobre el desarrollo de la clasificación (Inhelder y Piaget, 1964), el niño alcanza la capacidad para la clasificación jerárquica durante el período de las operaciones concretas. Los autores mantienen que es en este período donde el niño llega a «comprender» efectivamente las relaciones de inclusión jerárquica entre clases, comprensión que se evalúa a través del problema piagetiano de inclusión de clases.

En este problema se presenta al niño una categoría de objetos, por ejemplo flores, divisible en dos subclases mutuamente exclusivas, una de las cuales posee mayor número de elementos, por ejemplo 12 rosas y 6 tulipanes. Entonces se les pide comparar la extensión de la clase y la subclase mayor a través de preguntas del tipo: ¿hay más rosas o más flores? De acuerdo con la teoría de Piaget, el éxito en ese problema depende de la habilidad del niño para efectuar simultáneamente las operaciones reversibles de adición de clases (rosas + tulipanes = flores) y sustracción de clases (rosas = flores - tulipanes); o lo que es lo mismo, el niño debe considerar el todo (clase) al tiempo que mantienen la identidad de las partes (subclases). La comparación rosas-flores, le exige pensar en las rosas como rosas y como flores simultáneamente. Piaget encuentra que el niño no es capaz de operar en este 
sistema reversible y por tanto, de resolver el problema hasta aproximadamente los 7 u 8 años, cuando otras operaciones concretas ya se dominan. Antes de esta edad, el niño típicamente contesta: «hay más rosas» al hacer erróneamente la comparación simple entre subclases. Este hallazgo es acorde con la evolución encontrada en varios estudios directos de las clasificaciones de los niños (Vigotsky, 1962; Inhelder y Piaget, 1964; Bruner y col., 1966). Tales estudios sugieren que las clasificaciones de los niños atraviesan tres etapas generales: el niño muy pequeño, en un primer estadio, tiende a organizar los objetos considerando las relaciones temáticas que guardan entre sí; más tarde, en un estadio intermedio, es capaz de agruparlos basándose únicamente en sus semejanzas y diferencias, aunque los criterios son cambiantes; finalmente, hacia los 7 u 8 años, comienza a distribuir los objetos en clases estables y a organizarlos dentro de jerarquías lógicas.

Frente a esos datos, sin embargo, en los últimos años se ha aportado considerable evidencia de que incluso niños muy avanzados ya en el período de las operaciones concretas, fracasan en el problema clásico de inclusión. Estos niños, por ejemplo, frecuentemente cursan grados en los que ciertas tareas escolares parecen ser mucho más complejas que el problema de inclusión, lo cual sugiere que el fallo puede no deberse a la incomprensión de la lógica de la tarea, sino a algún déficit de procesamiento o ejecución (Winer, 1980). De hecho se ha constatado la influencia de numerosos factores de tipo perceptivo, lingüístico e incluso del contenido de las categorías de referencia, que pueden facilitar o dificultar de forma diversa la resolución del problema (ver la revisión citada de Winer y Alonso, Gutiérrez, 1986). Por otro lado, Markman (1978) ha demostrado que hasta aproximadamente los 11 años el éxito en esta versión clásica del problema, puede deberse a que el niño interpreta la mayor extensión de la clase respecto de la subclase como un hecho empírico (dado que se le facilitan este tipo de indicios) antes bien que como una consecuencia lógica de la inclusión, y que por tanto resuelva el problema basado en el conocimiento de tal hecho y no en la implicación lógica de la relación.
A la vista de los hallazgos precedentes, el problema piagetiano parece inadecuado como medida de la comprensión de las relaciones de inclusión - al menos en su versión clásica-, pues tanto los éxitos como los fracasos pueden deberse a factores ajenos a los requerimientos lógicos. Sin embargo, no conviene prescindir de esta tarea al estudiar el desarrollo de la comprensión de la inclusión, pues, en principio, no es descartable que una solución correcta esté basada en una auténtica comprensión de la asimetría, concretamente, en la apreciación de la extensión diferencial de las clases. Esta es la razón por la que hemos seguido utilizando la versión clásica en nuestro propio estudio, si bien, como forma más adecuada de evaluación, hemos introducido algunas modificaciones propuestas por Markman (1978) que evitan los éxitos basados en indicios de tipo empírico: bien omitiéndolos a partir de una presentación puramente verbal, bien a través de cuestiones adicionales en las que se hace la misma pregunta tras añadir o sustraer elementos a las clases, o bien refiriendo el problema a categorías totalmente nuevas para el niño. Además, considerando las numerosas variables en la presentación del problema que como se ha dicho facilitan o dificultan la solución y la ausencia de trabajos que evalúen su influencia de modo sistemático a lo largo del desarrollo (Winer, 1980), hemos realizado un control sistemático de las más representativas a saber: el contenido de clase frente al de colección; la presentación simplemente verbal de la tarea frente a la que añade elementos perceptivos y la referencia a categorías familiares frente a categorías nuevas. Estas tres variables recogen las fuentes de variación más generales y de efectos más probados en forma aislada.

En relación con la primera, tipo de estructura jerárquica de referencia, parece suficientemente demostrado por los trabajos de Markman (Markman y Siebert, 1976; Markman, 1973, 1978, 1980, 1981) que las colecciones constituyen una alternativa organizacional a las clases cuya estructura jerárquica «parte-todo» facilita la apreciación de la asimetría de la relación de inclusión en cuanto a la extensión diferencial de dos niveles jerárquicos. En este sentido, al incluir esta variable, tratamos de replicar su 
efecto al tiempo que lo analizamos en una perspectiva evolutiva amplia, lo cual no se ha hecho hasta ahora.

La segunda variable, referida a la modalidad de presentación, pretende recoger simultáneamente otros dos factores generales cuya influencia ha quedado patente en los trabajos revisados por Winer (1980): los de tipo perceptivo y lingüístico. Aunque se han presentado como dos condiciones simples de la misma variable, es posible el estudio de sus efectos por contraste, y además es presumible que varios aspectos particulares influyentes encontrados en estos dos tipos de variable, dependan de cada condición general tal y como nosotros las planteamos. Por lo demás, muchos de tales aspectos han sido considerados a partir del formato de las tareas, en los distintos planteamientos de las mismas cuestiones o bien se han distribuido al azar.

En cuanto a la tercera variable, tipo de categoría de referencia, ha sido considerada en tanto que su efecto se ha mostrado repetidamente tanto en las tareas de clasificación explícita como en los estudios indirectos sobre categorización; en estos trabajos la ejecución con categorías familiares es notablemente superior (ver la revisión de Alonso y Gutiérrez, 1986). Además, dada la naturaleza lógica de la tarea, constituye un buen modo de controlar -en la condición de categorías nuevas-, que la respuesta correcta se basa en la comprensión de la lógica del problema y no en la apreciación o memorización de un hecho empírico; procedimiento que, como ya se ha indicado, fue sugerido por Markman (1978).

De acuerdo con lo expuesto, nuestro primer experimento ha sido diseñado en orden a determinar la evolución de la comprensión de la extensión diferencial asimétrica entre clases, a partir del problema piagetiano que es el que específicamente incide en este aspecto. Como primera hipótesis 1.) Hemos establecido que la ejecución en este tipo de problema -o lo que es lo mismo, la comprensión de la extensión diferencial de clases-, mejorará con la edad, considerada independientemente de las demás variables en juego. Este pronóstico se apoya en la evidencia aportada por los numerosos trabajos experimentales realizados con versiones de la cuestión clásica, en los que las edades a las que se resuelve el problema son tan diversas como los propios estudios (Winer, 1980). Este hecho es el que nos lleva a rechazar que la apreciación de la extensión diferencial se alcance a una determinada edad como etapa del desarrollo y a suponer por el contrario, una adquisición progresiva desde las primeras edades.

Por otro lado, y atendiendo a las variables de planteamiento hemos postulado que:

2. Las ejecuciones con colecciones serán mejores que con «clases», tal y como sugieren los trabajos de Markman: la estructura de las colecciones resalta la asimetría de la relación inclusiva facilitando la apreciación de la extensión diferencial.

3. Las ejecuciones en las condiciones verbales serán superiores a las mostradas en las condiciones perceptivas. Cabe esperar este resultado dado que en estas últimas, el mayor número de la subclase en cuestión puede inducir una comparación distributiva por la evidente diferencia numérica, eligiéndose erróneamente tal subclase como respuesta. Esta hipótesis también es acorde con los datos aparecidos en la literatura sobre este punto.

4. La ejecución con categorías familiares será mejor que con categorías nuevas, supuesto que el conocimiento empírico pueda facilitar la respuesta correcta, lo cual también ha sido evidenciado repetidamente.

5. Las diferencias en ejecución respecto de las condiciones de las tres variables disminuirán progresivamente con la edad al incrementarse la comprensión de la lógica de la tarea, tal y como fue postulado en nuetra primera hipótesis.

Además, aparte de la posible interacción, cabe esperar.

6. un efecto aditivo en las combinaciones en que confluyan condiciones favorables o desfavorables de acuerdo con los efectos directos postulados (v. g. las condiciones «colección-verbal» serán más fáciles que las condiciones «clase-perceptiva»); a igualdad en alguna condición, las diferencias se producirán en la dirección del efecto principal de las variables restantes (v. g. se resolverá mejor «colección-perceptiva» que «claseperceptiva»); y en la alternancia de condiciones (v. g. «colección-perceptiva» frente a «clase-verbal») dependerá de la fuerza re- 
lativa del efecto favorable en las variables implicadas.

Análogas consideraciones cabe hacer respecto de las combinaciones triples e incluyendo asimismo la edad (delimitada en nuestro estudio a partir de los cursos escolares), en el sentido ya indicado de ir disminuyendo progresivamente las diferencias.

Finalmente cabe esperar.

7. Que estas tendencias generales queden moduladas por efectos interactivos varios, respecto de los cuales carecemos de base sobre la que proponer hipótesis concretas previas.

\section{Tarea y procedimiento}

Los problemas de tipo piagetiano tal y como aparecen en la prueba que se presenta en el anexo, se han elaborado a partir de la combinación sistemática de los tres factores bivariados ya señalados.

R. Organización jerárquica: clase $(C)$ v.s. colección (K).

S. Presentación: verbal (V) v.s. perceptiva $(\mathrm{P})$.

T. Categoría: familiar (F) v.s. nueva $(\mathrm{N})$.

Las 8 condiciones resultantes son, por tanto, en abreviatura: CVF, CVN, CPF, CPN, KVF, KVN, KPF, KPN. Cada una de ellas se ha establecido a partir de una presentación inicial en la que se exponen las categorías de referencia. Para las categorías nuevas se especifica la relación de pertenencia o inclusión (con categorías familiares se supone conocida aunque se ha aclarado en los casos dudosos). En las condiciones perceprivas la presentación incluye dibujos de algunos elementos de las subclases, siendo mayor su número en una de ellas, siguiendo la versión original de estos problemas. Para cada una de las condiciones se plantean cinco tipos de cuestiones que recogen otros aspectos influyentes observados en los trabajos previos:

1. Cuestión en la versión original clásica: «¿Hay más (clasè) o más (subclase)?». El orden de los términos de clase y subclase se ha distribuido al azar y la subclase referida ha sido siempre la mayor, siguiendo también aquí la versión original.

2. Cuestión en la versión facilitadora: «¿Qué quedará si quitamos (clase)?»
3. La misma formulación anterior referida a la subclase: «¿Qué quedará si quitamos (subclase)?». Esta cuestión induce la comparación distributiva entre subclases para hacerla contrastar con la colectiva, que es la que se requiere en las demás preguntas.

4. Cuestión en la versión clásica, previa adición de elementos a la subclase mayor: «Si se ponen dos (elementos de la subclase mayor), ¿habrá más (clase) o más (subclase mayor)?».

5. La misma formulación previa sustracción de elementos de la subclase menor: $« \mathrm{Si}$ se quita un (elemento de la subclase menor), ¿habrá más (clase) o más (subclase mayor)?» Estos dos últimos tipos de cuestiones eliminan la posibilidad de respuesta correcta en base a indicios empíricos ${ }^{2}$. El niño debe comprender que ni la adición ni la sustracción de elementos altera la relación de inclusión.

En las versiones de colección, los términos de clase han sido sustituidos por términos colectivos. En cada condición la cuestión primera se ha presentado siempre en primer lugar. La segunda, y la tercera, han seguido a la primera, si bien alternando aleatoriamente su posición relativa. Y en último lugar la cuarta y la quinta, también alternadas del mismo modo que en el caso anterior. Con este orden de presentación, hemos pretendido organizar el ejercicio en una secuencia coherente desde el punto de vista racional, al tiempo que las alternancias podían evitar en alguna medida la adquisición de hábitos de respuesta. Con cada cuestión, se han ofrecido las alternativas de respuesta también, en cada caso, en un orden azaroso por la misma razón.

Dado que las cuestiones se puntúan 1 ó 0 según sea la respuesta correcta o incorrecta, la puntuación máxima para cada condición ha sido de 5 y de $40(8 \times 5)$ para la prueba total.

\section{Análisis de datos}

Teniendo en cuenta que lo que tratamos de averiguar, en suma, es el efecto conjunto de varios factores en la combinación de sus condiciones respectivas, hemos considerado apropiado un análisis de varianza de las ob- 
servaciones obtenidas en la prueba, si bien se ha procedido previamente a una transformación de las mismas con el objeto de adecuarlas a este tipo de análisis. En concreto, como ya se ha indicado, las puntuaciones directas de cada sujeto en cada categoría de interacción resultaban de la suma de sus puntuaciones 1 ó 0 (según éxito o fracaso) en cada una de las cinco cuestiones referidas a la categoría. Dado que tales puntuaciones directas tienden a seguir una distribución binomial que no se ajusta a los supuestos del análisis de varianza, procedimos a su transformación en proporciones y posteriormente a la tansformación arco-seno de estas últimas. Como se sabe, el efecto de estas transformaciones es el de proporcionar conjuntos de nuevas puntuaciones relacionadas con las originales pero con varianzas homogéneas y con distribución normal o prácticamente normal, con lo cual, ha sido posible el ANOVA que pretendíamos.

El análisis se ha efectuado considerando todas las variables estudiadas: curso con 9 niveles desde preescolar hasta $8^{\circ}$, tipo de contenido (colección-clase), tipo de presentación (perceptiva-verbal) y tipo de categoría (nueva-familiar), las cuales han dado lugar a un diseño de $9 \times(2 \times 2 \times 2)=72$ condiciones, con «medidas repetidas" de las incluidas entre paréntesis (en cada curso cada sujeto recibe las 8 condiciones). En los casos en los que se ha alcanzado el nivel de significación $(\mathrm{p}<0.05$ ó $\mathrm{p}<0.01)$ se ha aplicado la prueba de Tukey para determinar entre qué grupos era significativa la diferencia de medias, prueba que se ha escogido dado que se adecua a nuestro caso al ser igual el número de observaciones en cada categoría.

\section{Resultados y discusión}

La tabla I presenta los resultados del análisis de varianza efectuado, que en general son acordes con nuestras hipótesis. Los efectos principales son significativos $(p<0.01)$ en las variables curso $(C)$, tipo de contenido $(\mathrm{R})$ y tipo de categoría de referencia ( $T)$ pero no llega a serlo el tipo de presentación (S). Así mismo se dan efectos de interacción significativos entre las variables RC, RS, RT, RTC, RSTC.

En cuanto al efecto de la variable curso, como muestra la gráfica 1 se da un incremento regular $y$ constante desde preescolar a $8^{\complement}$ en la comprensión de la extensión diferencial entre clases. Las diferencias sólo son significativas entre cada grupo y el que le sigue en segundo, tercero o cuarto lugar (ver tabla II), lo cual parece lógico si se tiene en cuenta la variabilidad interindividual. Este resultado, obtenido considerando el conjunto de las cuestiones, es contrario a las afirmaciones de Inhelder y Piaget (1964) acerca del desarrollo de la habilidad para evaluar las diferencias de extensión entre clases, pues a la edad de 7-8 años en que este autor sitúa la comprensión plena de este aspecto, no se da el salto brusco que cabía esperar. Aunque a partir de $3^{0}$ (8-9 años), parece incrementarse la pendiente de la curva, las diferencias no son significativas; el progreso es por tanto regular sin alcanzarse el techo en ningún punto, lo cual es acorde con nuestra primera hipótesis.

En relación al tipo de estructura jerárquica implicada $(\mathrm{R})$, nuestra hipótesis tambien queda confirmada: la ejecución es significativamente superior en las cuestiones referidas a colecciones $(\mathrm{K})$ frente a las que se refieren a clases $(\mathrm{C})(\overline{\mathrm{K}}=1.28 ; \overline{\mathrm{C}}=.99 ; \overline{\mathrm{K}}-\overline{\mathrm{C}}$ $=.29 ; \mathrm{p}<.001)$. Este efecto sin embargo, está modificado por las otras tres variables estudiadas:

Interacción $R C$. Como habíamos previsto las diferencias en la ejecución con clases y colecciones disminuyen progresivamente con la edad, tal y como se refleja en la gráfica 2, aunque en todos los cursos son significativas $(\mathrm{p}<0.01)$ excepto en $8^{\circ}$ (ver tabla III); este hecho sugiere una probable estabilidad a partir de este nivel alrededor del techo de la prueba. Considerando ambas condiciones por separado, se observa que el incremento con la edad en el caso de las clases, sólo es significativo entre cada grupo y el que le sigue en tercer lugar, no existiendo diferencias significativas, a partir de $6^{\circ}$, entre este grupo y los siguientes; en el caso de las colecciones, sólo es significativa la diferencia entre un curso y el que le sigue èn cuarto lugar, desapareciendo la significación ya a partir de $4^{0}$ entre éste y los siguientes. Así pues parece, como cabía esperar, que la estabilidad en la ejecución con colecciones se alcanza más pronto $\left(4^{Q}\right)$ que con clases $\left(6^{\circ}\right)$. 
- Interacción $R S$. Las diferencias entre clases y colecciones también se ven afectadas por el modo de presentación de la tarea. Como puede apreciarse en la gráfica 3 , la presentación perceptiva acentúa el efecto, fundamentalmente porque en el caso de los problemas con clases, esta presentación perceptiva empeora significativamente la ejecución frente a la presentación verbal, lo cual no ocurre en el caso de los problemas con colecciones, donde la tendencia parece ser la contraria. En relación con este resultado, es curioso constatar que, en contra de nuestra hipótesis, no se da efecto principal significativo del modo de presentación (tabla 1; F de V: S; $\mathrm{p}=0.3996$ ), es decir, que nuestra hipótesis sobre el efecto favorable de la presentación verbal frente a la percepriva sólo se cumple en el caso de que el problema se refiera a clases. Este hecho tiene, sin embargo, una explicación clara: la hipótesis se basaba en la consideración de que la presentación perceptiva podía inducir fácilmente la comparación errónea entre subclases ante la evidente diferencia numérica, sin considerar la clase o el «todo» mayor formado por ambas subclases. Si como se ha argumentado, la estructura de las colecciones resalta la relación parte-todo, ésta se verá mucho menos afectada por la circunstancia numérica o incluso podría hacerla más evidente, al concretar el «todo empírico» que constituyen las colecciones (frente al abstracto de las clases). Así se explicaría tanto el efecto claro en las clases como la tendencia observada en las colecciones.

- Interacción $R T$. El tipo de categoría de referencia ha afectado a los problemas de clases y colecciones en forma semejante al modo de presentación. Como puede verse en la gráfica correspondiente (gráfica 4) la referencia a categorías conocidas mejora significativamente la ejecución en los problemas de clases mientras que no tiene efecto significativo en las cuestiones de colección, incrementando así el contraste entre estas dos condiciones de la tarea. Sin embargo, en este caso no se produce la tendencia a la inversión de efectos observada en el caso anterior. La explicación de estos resultados también es sencilla: las categorías, en tanto que clases, sólo afectan los problemas en que estas clases se manejan directamen- te, resultando más fáciles si son familiares que si son nuevas tal y como se apuntaba en nuestra hipótesis. Cuando se refiere la clase mayor con un término colectivo es indiferente que las demás aludan a categorías nuevas o familiares puesto que la base de la facilitación no queda alterada. Sin embargo, independientemente de la incidencia en la evaluación lógica del problema - dificultada en un caso, inalterada en otro-, cabe esperar un mejor manejo del problema en términos familiares que en términos desconocidos, como puede esperarse en cualquier otro tipo de problema. Esto es lo que explicaría la no inversión de efectos y el hecho de que el efecto principal de este tipo de variable sí resulte significativo (Tabla $1 ; \mathrm{F}$ de $\mathrm{V}: \mathrm{T} ; \mathrm{p}=0.000<0.001)$, todo lo cual está en la línea de nuestras hipótesis.

- Interacción TC. El mismo tipo de argumento explica asi mismo el hecho de que esta variable no interactúe con la edad: independientemente de ésta y de cualquier otro tipo de condición, lo familiar se procesará más fácilmente que lo nuevo. Sin embargo, como apuntábamos en nuestra hipótesis 4, también es esperable que con la edad, al aumentar la capacidad de procesamiento en general, y en nuestro caso particular, la comprensión de la lógica de clases, el contraste entre lo familiar y nuevo disminuya. La tendencia que reflejan los datos no es exactamente ésta: la discrepancia se acentúa en las edades medias pero no en las extremas. Esto también es razonable: el problema en los primeros cursos resulta tan difícil en sí mismo que los términos familiares poco pueden facilitarlo; y a la inversa, en los últimos cursos el problema resulta fácil incluso con términos nuevos; en los cursos centrales donde la dificultad es media, la facilitación de los términos familiares encuentra campo de acción y así tiende a reflejarse (ver gráfica 5).

- Interacción RTC. En línea con las interpretaciones precedentes cabe explicar el efecto interactivo RTC que en este caso sí resulta significativo. Con colecciones, sigue siendo escaso o nulo el efecto del tipo de categoría en todas las edades. Con clases vuelve a minifestarse la tendencia vista en el caso de la interacción TC, aunque sólo se da en $5^{\circ}$ una diferencia significativa $(\overline{\mathrm{CF}}-\overline{\mathrm{CN}}$ $=.26 ; \mathrm{p}<0.01)$. Así pues en suma, la facili- 
tación de las categorías familiares sólo se produce en los problemas con clases dentro de las edades intermedias, cuando la dificultad del problema también es media.

- Interacción RSTC. La interacción RST considerada globalmente no es significativa, pero sí lo es en relación con la edad (ver tabla IV). Probablemente RST no es significativa debido a efectos compensatorios de la dificultad de cada condición entre todas las edades: nótese que las 8 combinaciones siguen la misma pauta creciente desde preescolar a $8^{0}$ donde las diferencias se hacen mínimas; esta tendencia hace que las medias en cada condición ya se acerquen hasta el punto de no ser significativamente diferentes. Sin embargo, considerando las combinaciones en cada edad puede apreciarse que siguen en general la dirección de los efectos ya explicados. En suma, que las colecciones se manejan mejor que las clases en todas las edades, disminuyendo las diferencias progresivamente; que lo perceptivo frente a lo verbal y lo nuevo frente a lo familiar afecta poco a las colecciones, alternándose la calidad de sus efectos en forma más o menos aleatoria a lo largo de todas las edades; que las categorías familiares mejoran la ejecución con clases sobre todo en las edades medias; y que la presentación perceptiva empeora esta ejecución, aunque se suaviza si coincide con categorías familiares y acentúa con categorías nuevas. Además, en cinco de los grupos de edad se observa el efecto aditivo combinado de los tres factores analizados en la dirección prevista: $\mathrm{KPF}>\mathrm{CPN}$.

\section{EXPERIMENTO 2: INFERENCIAS TRANSITIVAS; EVOLUCION DE LA TRANSITIVIDAD Y ASIMETRIA DE LA TRANSITIVIDAD}

\section{Introducción}

Ante las limitaciones de las tareas de clasificación como vía de acceso al conocimiento conceptual del niño, los investigadores han utilizado procedimientos más indirectos estudiando, por ejemplo, la manipulación espontánea de objetos o la organización de materiales en la memoria. Las tareas propuestas en estos estudios requieren un conocimiento mucho menos explícito que el procedimiento de clasificación tradicional y contienen pocas demandas adicionales; como consecuencia la ejecución mejora notablemente. Pero los resultados, al igual que en las tareas de clasificación, han de interpretarse con cautela, pues las relaciones de categorización pueden ser fácilmente confundidas con otro tipo de relaciones (Markman, 1984). Así, las ejecuciones en este tipo de tareas tampoco permiten una evaluación explícita de la habilidad del niño para organizar jerárquicamente los conceptos.

Existen, sin embargo, otras medidas más directas de esta habilidad según criterios de asimetría y transitividad. Este es el caso del trabajo de Keil $(1977,1979)$ sobre el desarrollo del conocimiento ontológico (categorías básicas de lo existente). Sus resultados sugieren que incluso niños de sólo 5 años son capaces de hacer uso -implícito al menos- de un esquema de clasificación jerárquico con relaciones de inclusión asimétricas y transitivas. No obstante, hay que considerar que tal conocimiento se expresa en propiedades muy generales (tener color, peso, duración...) atribuidas también a categorías muy generales (objetos físicos, sucesos, ideas...), caso particular que no acontece frecuentemente. en el manejo de jerarquías ordinarias de categorías más restringidas (cachorro-perro-animal-etc.); la generalización, por tanto, puede no estar justificada (Markman, 1984).

Las medidas más explícitas de la comprensión de la asimetría y transitividad de la inclusión, son las empleadas en los estudios de Harris (1975) y Smith (1979) a través de problemas inferenciales, siendo el de Smith el que aporta evidencia más clara de la apreciación de la lógica de la inclusión por parte del niño. Este autor examina niños de 4 a 7 años mediante problemas en los que, tras presentar una relación de inclusión en la que la subclase es desconocida para el niño, se le pide juzgar la veracidad de una inferencia transitiva de tal subclase en referencia a una tercera categoría. En un caso la inferencia debe realizarse sobre la pertenencia de clase según planteamientos del tipo: «Un _ es una clase de X, ¿un _ tiene que ser un $Y »$, incluyéndose en el espacio en blanco un término real que el niño 
no conoce. Este problema se presenta en tres versiones: cuando ia inferencia es válida (v.g. «Un pug es una clase de perro, ¿un pug tiene que ser un animal?») indeterminada (v.g. un pug es un animal, ¿un camión tiene que ser un garaje?»). En otra formulación la inferencia debe efectuarse sobre propiedades de clase; el planteamiento es análogo: "Todos los Xs tienen —; ¿todos los Ys tienen que tener __?». De nuevo el problema tiene una versión válida (v.g. «Toda la leche tiene lactosa, ¿todo el chocolate con leche tiene que tener lactosa?») indeterminada («Toda la leche tiene lacto$\mathrm{sa}$, ¿todas las bebidas tienen que tener lactosa?») e inválida (v.g. "Toda la leche tiene lactosa, ¿todas las zapatillas tienen que tener lactosa?»).

Los datos obtenidos por Smith indican que, en general, los más mayores (6-7 años) realizan adecuadamente estas inferencias transitivas de pertenencia y propiedades y que aproximadamente el $65 \%$ de los niños de 4 años también responden de forma correcta. Estos resultados nos han llevado a incluir también este tipo de problemas inferenciales en nuestro estudio, dado que, por una parte, implican directamente el manejo de la transitividad y asimetría de la inclusión y por otra, hasta los niños más pequeños los resuelven en alguna medida. No obstante el procedimiento original se ha modificado en varios puntos:

En primer lugar, aunque también se han propuesto dos conjuntos de cuestiones para incluir los dos tipos de inferencia planteados por Smith, la formulación se ha simplificado: la inferencia relativa a la pertenencia o inclusión de clase, se ha presentado según la siguiente formulación: «Un _ es un $\mathrm{X}$, ¿un - es un $\mathrm{Y}$ ?»; de igual forma la inferencia relativa a propiedades características de estas clases, se ha presentado con una formulación simplificada análoga: «Todos los Ys tienen — ¿todos los Xs tienen _ ?». Esta leve variación en el planteamiento se ha introducido para evitar la expresión «tener que», atendiendo la sugerencia del propio Smith (1979), sobre la probable confusión que podría dar lugar dada su connotación de «necesidad».

Por otro lado, el término de clase o de propiedad sobre el que se plantea la inferencia ha sido inventado en todos los casos (procedimiento de Harris, 1975), para asegurar que aquella se realiza sobre una base lógica y no sobre un posible conocimiento empírico.

Además, hemos eliminado las versiones inválidas (clases $\mathrm{X}$ e $\mathrm{Y}$ no relacionadas) para ambos tipos de problemas, por considerar que estas formas de planteamiento no recogen nada nuevo en cuanto a la comprensión de la inclusión y, por el contrario, pueden constituir un elemento de confusión al sugerir la posibilidad de una relación en realidad inexistente, que podría contaminar la evaluación de otras cuestiones en las que sí se da o puede darse la relación inclusiva. Es decir, que sólo se han mantenido las versiones válidas ( $\mathrm{X}$ incluida en $\mathrm{Y}$ ), que requiere la comprensión de la transitividad, e indeterminadas ( $\mathrm{Y}$ incluida en $\mathrm{X}$ ), que requiere la comprensión de la asimetría de la transitividad. En relación con este tipo de cuestiones, la literatura revisada por Evans (1982), sugiere la mayor dificultad de la asimetría de la transitividad, dado que en ella están implicados ambos aspectos lógicos de la inclusión; este hecho también ha sido señalado por Markman (1984) y así lo recogemos en una de nuestras hipótesis.

Finalmente, en las cuestiones de transitividad de pertenencia se ha añadido un planteamiento paralelo en forma negativa, y tanto los de pertenencia como los de propiedades se han formulado relativos alternativamente a categorías nuevas y familiares. El objeto de estas variaciones ha sido el de evaluar también respecto a este tipo de problemas la influencia diferencial de las dificultades adicionales de procesamiento que pudieran suponer. La formulación negativa se ha introducido teniendo en cuenta la dificultad adicional que parece conllevar el procesamiento de la información negativa (Evans, 1982).

Con las anteriores modificaciones y las nuevas variables introducidas, los problemas inferenciales han concretado nuestro segundo experimento, en el cual se examina la evolución de la comprensión de la transitividad y asimetría de la transitividad de la inclusión. Como en el experimento anterior nuestra primera hipótesis 1) establece la ejecución - manejo de la transitividad- mejorará con la edad considerada aparte de las demás variables, debido al pro- 
greso en la comprensión de este aspecto lógico. Atendiendo a las otras variables y en base a las justificaciones ya apuntadas, hemos formulado las siguientes hipótesis:

2)- el éxito será mayor con las cuestiones qeu requieren la evaluación de la transitividad simple, frente a aquellas que requieren valorar la asimetría de la transitividad ya que, como se ha señalado, en estas últimas están implicados ambos aspectos de la inclusión.

3) - la ejecución será mejor cuando la formulación de las cuestiones sea positiva frente a la negativa por las razones ya señaladas.

4) - la ejecución con categorías familiares será mejor que con categorías nuevas.

5) - es de esperar, asimismo, que las anteriores diferencias vayan disminuyendo conforme avanzan los cursos al ir incrementándose la habilidad lógica como tal.

En este experimento tampoco pueden hacerse predicciones sobre los posibles efectos interactivos que pueden modular los efectos directos postulados, excepto en la dirección de su suma. Las consideraciones que cabe hacer en este punto son análogas a las expuestas para el experimento 1 .

\section{Procedimiento}

La combinación de las distintas condiciones implicadas en este segundo experimento ha dado lugar a 12 cuestiones en total, 8 que requieren una inferencia sobre pertenencia $(\mathrm{Pt})$ y 4 que plantean inferencias sobre propiedades $(\mathrm{Pr})$. La mitad en cada grupo requería la evaluación de una inferencia transitiva directa $(T)$ y la orra mitad, la comprensión de la asimetría de la transitividad (A). La mitad estaban referidas a categorías nuevas (N) y la otra mitad a categorías familiares (F). Además, 4 de las cuestiones de pertenencia tienen una formulación positiva $(+)$ y las otras 4 negativa $(-)$. El diseño resultante ha sido, por tanto, en el caso de inferencias de pertenencia $(\mathrm{Pt})$ : 2 requerimientos lógicos $(A-T) \times 2$ tipos de categorías de referencia $(\mathrm{F}-\mathrm{N}) \times 2$ tipos de formulacion (+ó - ) $=2 \times 2 \times 2=8$ condiciones. $Y$ en el caso de inferencia sobre propiedades, sin la última variable, $2 \times 2=4$ condiciones.
Como estas condiciones se han concretado en cuestiones individuales puntuadas 0 ó 1, las puntuaciones globales han sido como máximo de 8 y 4 respectivamente.

\section{Análisis de los datos}

Dada la desigualdad del diseño presentado y el tipo de puntuación (0-1) para cada combinación de variables, se ha desestimado el ANOVA, dado que aquellas no cumplían los requisitos de dicho análisis, requisitos que no era posible conseguir ni aun transformando las puntuaciones. En relación con este punto queremos señalar, no obstante, que la necesidad de no alargar excesivamente el examen de los niños, dados nuestros objetivos iniciales, nos aconsejó no incrementar el número de ítems en esta parte del experimento, aunque ello fuese en detrimento de la posibilidad de utilizar un análisis más elegante de nuestros resultados. En consecuencia se ha procedido a comparaciones simples entre grupos de sujetos y grupos de cuestiones (referidas a una u otra condición por separado) a través de pruebas de $t$ de diferencia de medias.

\section{Resultados y discusión}

En relación con nuestra primera hipótesis, hemos encontrado que, en efecto, la habilidad para manejar la transitividad de la inclusión crece con la edad. Como muestra la gráfica conjunta 6 , la pauta de crecimiento respecto de la transitividad de pertenencia es semejante a la que se muestra en la transitividad de propiedades, lo cual sugiere que estos dos aspectos no son sustancialmente diferentes y que lo que caracteriza a ambos es la misma lógica de transitividad implicada. Así, la curva de crecimiento global resulta paralela a las anteriores como mera suma.

De $4^{9}$ a $6^{9}$ curso parece darse un cambio notable, ya que a partir de este momento las diferencias de los cinco primeros cursos con los siguientes son significativas $(p<0.01$ en todos los casos). Cabe destacar el resultado extraño obtenido en $1^{0}$, significativamente más bajo que el observado ya en $2^{o}$ e incluso que el de preescolar en el 
caso de transitividad de propiedades; resultado para el que no tenemos explicación aparte de la posibilidad de que se deba a algún factor de azar.

- Como puede apreciarse en la gráfica 7 y de acuerdo con nuestra $2^{a}$ bipótesis, los problemas de transitividad simple se resuelven con mayor éxito que implican asimetría de la transitividad en todas las edades, aunque en $3^{\circ}, 5^{\circ}$ y $8^{\circ}$ la diferencia no es significativa (ver tabla $V$ ). También se observa una pauta creciente en las cuestiones que implican asimetría, y en consecuencia una cierta tendencia a la disminución de las diferencias conforme avanza el curso. Precisamente la falta de significación en los grupos de $3^{9}, 5^{\circ}$ y $8^{\circ}$ puede interpretarse como efecto de esta tendencia. Esta se muestra mucho más clara con los problemas de propiedades, como puede verse en la gráfica 8 ; las curvas son muy semejantes a las anteriores, si bien la diferencia en $5^{\circ}$, que en el caso de la pertenencia no era significativa, aquí sí lo es y en $8^{\circ}$ la ejecución es idéntica (ver tabla VI). En cualquier caso los datos muestran que la dificultad de la asimetría de la transitividad es mayor que la de la simple transitividad.

- En las cuestiones de pertenencia la ejecución diferencial en cuanto al tipo de formulación - positiva o negativa- del problema, punto al que se hace referencia en nuestra $3^{a}$ hipótesis, es menos marcada pero va en la dirección prevista (ver gráfica 9): los problemas planteados en forma positiva son más fáciles en todas las edades aunque la diferencia sólo es significativa en $4^{9}$ y en $6^{\circ}(\mathrm{p}<0.05)$. La pauta de progreso con el curso también es menos pronunciada y más paralela en las dos condiciones: la cuantía de las diferencias se mantiene muy semejante en todos los cursos; no parece reducirse con la edad. Esto es lógico dado que ya desde los primeros cursos la ejecución no es muy diferenté y por tanto, prácticamente no existe margen sobre el que pueda producirse un acercamiento. No obstante, el hecho de que la ejecución en los problemas formulados negativamente sea sistemáticamente inferior a los formulados de manera positiva, está en línea con los resultados de los trabajos con adultos revisados por Evans (1982) a los que ya hemos hecho referencia.
- En relación con el efecto del tipo de categoria de referencia - nueva o familiar-, el patrón aparecido es bastante similar: las categorías familiares mejoran la ejecución en todas las edades y la curva de crecimiento es muy poco pronunciada y paralela respecto de ambas condiciones (ver gráfica 10). Aquí, sin embargo, curiosamente aparecen las diferencias significativas en os cursos extremos (ver tabla VII). Este efecto es inverso al producido por esta misma variable en los problemas piagetianos con términos de clase. Como se recordará lo familiar sólo favorecía la ejecución en las edades medias; en los extremos no tenía efectos significativos, lo cual atribuíamos a la gran dificultad del propio problema en los primeros cursos y su extrema facilidad en los últimos, de modo que en ambos casos no podía apreciarse el efecto facilitativo de lo familiar. En el caso que nos ocupa, respecto de los problemas de transitividad de pertenencia ocurre, como decíamos, exactamente lo contrario: las categorías familiares muestran un mayor efecto facilitativo en los primeros y últimos cursos. En los primeros el efecto es el esperado pero no en los últimos; nos inclinamos a pensar que en estos últimos cursos las diferencias significativas son producto del azar y que, en realidad, la diferencia en ejecución con categorías nuevas y familiares, siendo apreciables al principio, tienden a reducirse con el curso, apareciendo efectos mínimos ya a partir de $3^{\circ}$. Esto es lo que estaría de acuerdo con nuestra hipótesis. En cualquier caso, el efecto de esta variable es muy leve y a juzgar por la curva aparecida con los problemas de propiedades, tal vez debería despreciarse en las dos áreas. En el caso de los problemas de propiedades aparecen valores cruzados y sólo se da una diferencia significativa al $5 \%$ en $7^{\circ}$. Así pues, cabe concluir que las categorías familiares facilitan poco el tipo de problemas que tratamos a partir de $3^{0} \mathrm{de}$ EGB, quizá -análogamente a lo ocurrido con las cuestiones piagetianas en términos de clase-, porque su gran dificultad intrínseca desborda su débil efecto. Téngase en cuenta que en estos problemas el techo de la prueba no se ha alcanzado: en los problemas de pertenencia las puntuaciones globales más altas están en torno a 3.5 siendo 8 la máxima posible; en las cuestiones de 
propiedades las más altas están alrededor de 2.5 sobre una máxima de 4 .

Por lo que se refiere a la interacción entre variables, los resultados tienden a ir en la línea de lo señalado, fundamentalmente en los primeros cursos ( $\mathrm{T}+\mathrm{F}>\mathrm{A}-\mathrm{N})$.

Como resumen, cabe apuntar que el efecto de la edad es el esperado: la ejecución con los problemas de transitividad mejora progresivamente con cada curso escolar con un progreso más marcado de $4^{\mathrm{Q}}$ a 6 ; ; y puede esperarse que el éxito siga creciendo en sujetos de mayor edad que la de los estudiados. Se ha evidenciado igualmente que los problemas de simple transitividad son más fáciles que aquellos que implican también asimetría, aunque su dificultad tiende a igualarse a medida que aumenta el nivel escolar. La formulación positiva facilita el problema si bien su efecto es poco marcado. Esto mismo ocurre con referencia a categorías familiares frente a las nuevas; en este caso la facilitación de las categorías familiares podría incluso despreciarse. Finalmente, no parece haber diferencia entre los plateamientos relativos a la pertenencia de clase y los relativos a sus propiedades, probablemente porque la lógica implicada es la misma.

\section{EXPERIMENTO 3: APLICACION DE CUANTIFICADORES; EVOLUCION DE LA COMPRENSION DE LA ASI- METRIA SIMPLE DE PERTENENCIA}

\section{Introducción}

Además de los problemas inferenciales Smith (1979) presenta al niño cuestiones de inclusión cuantitativa a través de los cuantificadores «todos» $y$ «algunos» en la forma "¿Todos los Xs son Ys?» y « ¿Algunos $\mathrm{Xs}$ son $\mathrm{Ys}$ ?». En unos casos $\mathrm{X}$ es una subclase de $\mathrm{Y}$ (v.g. $\mathrm{X}=$ peras e $\mathrm{Y}=$ fruta); en otros una clase supraordinada (v.g. $\mathrm{X}=$ animal e $\mathrm{Y}=$ perros); $y$ en otros $\mathrm{X}$ e $\mathrm{Y}$ son categorías no relacionadas.

En este nuevo tipo de tarea Smith encuentra que en la mayoría de los casos incluso los niños de 4 años interpretan la inclusión en forma asimétrica, resultado que es contrario al encontrado en los primeros estudios de Inhelder y Piaget (1964) quie- nes ya utilizaron el mismo tipo de cuestiones. Considerando estos datos y dado que en tales cuestiones claramente se maneja la asimetría simple de inclusión o pertenencia, parece evidente la utilidad de las mismas para examinar la habilidad del niño en este aspecto lógico de la inclusión. Con esta certeza se ha utilizado también aquí este tipo de tarea, la cual constituye el tercer y último experimento con el que completamos nuestro estudio. En este caso no se ha modificado el procedimiento original, si bien hemos seguido prescindiendo de la version inválida, en la que las clases implicadas no están relacionadas. Sólamente se ha ofrecido una versión verdadera ( $X$ es una clase de $\mathrm{Y}$ ) y otra falsa ( $\mathrm{Y}$ es una clase de $\mathrm{X})$ para los dos cuantificadores según la forma: «¿Todos los Xs son Ys?» y «¿Sólo algunos Xs son Ys?». Además, las cuatro cuestiones se han presentado tanto en referencia a categorías conocidas como a categorías nuevas, a fin de seguir evaluando el efecto de esta variable que en este caso ha sido la única viable.

Al igual que los experimentos precedentes y por las mismas razones se esperaba que:

1) la ejecución mejorase con li edad

2) las cuestiones referidas a categorías familiares resultasen más fáciles iue las referidas a categorías nuevas

3) que las diferencias fuesen disminuyendo con la edad.

\section{Tarea y procedimiento}

De acuerdo con lo ya expuesto, han resultado 4 cuestiones para cada uno de los cuantificadores: 2 referidas a categorías nuevas y otras 2 referidas a categorías familiares; y cada una de ellas en versión verdadera y falsa. Como en tareas anteriores se ha puntuado 1 ó 0 el éxito o el fracaso respectivamente, en el juicio de cada proposición. Así pues ha sido 4 la puntuación máxima en cada bloque y 8 la máxima total.

\section{Análisis de datos}

El diseño, aunque más secillo, es semejante al del experimento anterior y con las 
mismas características; así que se ha analizado de igual forma mediante comparaciones simples con pruebas de $t$.

\section{Resultados y discusión}

Los resultados en este tercer experimento muestran otra vez el efecto de la edad: la gráfica 11 muestra el desarrollo encontrado al avanzar los cursos escolares. Como en los problemas de transitividad, el progreso más notorio se produce en los cursos intermedios, de $4^{\circ}$ a $6^{\circ}$, aunque aquí la pendiente es más suave.

En relación con el efecto del tipo de categoría, los datos evidencian en este caso una influencia muy marcada (ver gráfica 12); en todos los casos las cuestiones con categorías familiares se resuelven con mayor éxito que las referidas a categorías nuevas, diferencia que además resulta significativa en todos los grupos (ver tabla VIII). Por el contrario, las diferencias respecto de cada condición entre los diversos cursos son relativamente pequeñas. En las cuestiones con categorías nuevas la evolución es lenta y las diferencias sólo llegan a ser significativas respecto de los últimos cursos, especialmente con $7^{\circ}$ ( $p<0.01$ en todos los casos). Con categorías familiares la evolución es más irregular pero se alcanza un nivel muy alto ya a partir de $4^{Q}$, dándose en $6^{Q}$ una ejecución prácticamente perfecta.

Así pues, en las cuestiones de cuantificadores la referencia a categorías familiares tiene un claro efecto facilitador, efecto que se manifiesta mucho más débil en los otros problemas estudiados. La explicación sigue en la línea de lo ya argumentado en relación con estas tareas previas. En ellas el efecto se debilitaba más cuando más dependía el éxito de la comprensión puramente lógica del problema (con términos de clase en las cuestiones piagetianas y generalizadamente en los problemas de transitividad). En los problemas con cuantificadores los requerimientos lógicos pueden perderse totalmente cuando las relaciones se establecen con categorías familiares ya que, en este caso, el puro conocimiento empírico es suficiente para responder de forma correcta; mientras que las categorías nuevas sí exigen la evaluación lógica del problema. Así, las diferencias encontradas entre los grupos quizá son sólo de conocimiento en el primer caso (categorías familiares) mientras que lo son de grado de comprensión de la relación de inclusión en el segundo (categorías nuevas).

\section{COMPARACIÓN ENTRE LOS TRES TIPOS DE TAREAS}

Al objeto de precisar el orden de adquisición de los distintos aspectos de la inclusión implicados en las tareas estudiadas, hemos comparado entre sí los resultados globales obtenidos en cada tipo de problema dentro de cada curso. Para ello se ha procedido previamente a la transformación de las puntuaciones directas en proporciones (tabla IX) —dado que el número de cuestiones en cada caso ha sido diferente-, y se ha hallado la significación de las diferencias entre las mismas. La gráfica 13 presenta conjuntamente la evolución encontrada en cada uno de los procedimientos y la tabla X la significación de las diferencias de proporciones.

Como puede apreciarse, la ejecución con los problemas piagetianos y de cuantificadores progresa de forma paralela (no existen diferencias significativas excepto en $8^{Q}$, resultado que puede deberse a efectos de muestreo), si bien estos últimos parecen tener un dificultad levemente superior: excepto en preescolar y $1^{Q}$ la curva siempre va por debajo de la correspondiente a las cuestiones de extensión diferencial. Por otro lado, ambos tipos de tareas aparecen significativamente más fáciles que los problemas de transitividad en todas las edades y al $1 \%$. Este es un resultado sorprendente dado que en los primeros estudios de $\mathrm{Ha}$ rris (1975) y Smith (1979) eran este tipo de problemas los que los niños más pequeños podían resolver, en contraste con la gran dificultad de las cuestiones piagetianas. En estos trabajos los niños de 5 a 7 años son capaces de hacer correctamente las inferencias transitivas. En nuestro estudio se evidencia, por el contrario, que los problemas inferenciales de transitividad son los más difíciles en todas las edades (5-15 años), no habiéndose alcanzado en ninguna de ellas la comprensión plena de este as-

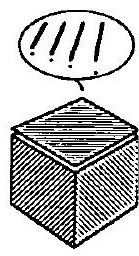


pecto lógico de la inclusión. Es posible que este resultado se deba fundamentalmente a alguna de las modificaciones del procedimiento y a la amplia perspectiva que proporciona un estudio evolutivo en edades muy diversas. En relación a lo primero hemos de resaltar el hecho de que en los planteamientos que nosotros hemos utilizado, el término al que se refería la inferencia siempre era desconocido en tanto que inventado, y, por consiguiente, en todos los casos era obligada la evaluación lógica del problema eliminándose completamente la posibilidad de respuestas correctas en base a un conocimiento previo. Quizá sea esta naturaleza estrictamente lógica del problema, la responsable de la mayor dificultad encontrada en nuestro trabajo.

\section{CONCLUSIONES}

Nuestro estudio ha puesto de manifiesto que la comprensión de la inclusión se desarrolla con la edad en una secuencia progresiva regular y constante. Esto es especialmente cierto en la comprensión de la exrensión diferencial y la aplicación correctamente asimétrica de los cuantificadores. La comprensión de la transitividad parece dar un salto notable entre los 9 y 12 años, pero incluso a partir de esta edad continúa el progreso.

En relación con las diversas condiciones de las tareas, se ha confirmado que el manejo de la asimetría de la inclusión es más fácil en relación a una estructura simple de colección que en referencia a la estructura de clases; en esté sentido constituye una adquisición más temprana, y consistentemente menos vulnerable, a los efectos de variables de planteamiento de las tareas. En términos de clases, la apreciación de la extensión diferencial y asimétrica se ve dificulta- da al presentar la tarea con elementos perceptivos que inducen representaciones erróneas y al hacer referencia a categorías desconocidas que impiden el apoyo en un conocimiento previo. Las categorías familiares, sin embargo, facilitan muy poco la comprensión de la transitividad de la inclusión, especialmente la asimetría de esta relación, si los términos sobre los que se infiere sí son nuevos, debido a que la naturaleza lógica del problema queda inalterada; por esta misma razón la formulación negativa no incrementa demasiado la gran dificultad que ya posee el problema en sí mismo: su resolución es deficiente incluso en los últimos niveles de EGB. En consonancia con estos datos, la aplicación de cuantificadores ya empieza a ser correcta en los primeros cursos cuando las categorías relacionadas son conocidas.

En general, los resultados obtenidos ponen de manifiesto que las condiciones de la tarea limitan en forma diversa la habilidad que el niño es capaz de desarrollar, al añadir dificultades de procesamiento a los requerimientos estrictamente lógicos. En relación con este hecho y en línea con las conclusiones integradoras de Gelman v Baillargeon (1983), se ha puesto de mani.iesto que las habilidades de inclusión no sosı una cuestión de todo o nada, sino que en todos sus componentes se da un considerable desarrollo con la edad. Cierta habilidad ya está presente desde las primeras edades estudiadas, aunque sólo se exprese bajo condiciones favorables; cuando se desarrolla la capacidad de procesamiento de información, aumenta el conocimiento sobre el mundo y las estrategias perceptivas y cognitivas del niño se hacen más eficientes, también mejora la comprensión de las relaciones de inclusión, y en consecuencia, la habilidad para detectar y hacer uso de estructuras clasificatorias de inclusión jerárquica, también se desarrolla. 
Efecto de las variables curso, tipo de organización jerárquica (clase o colección), tipo de presentación (verbal o perceptivo-verbal) y tipo de categoría (familiar o nueva) en la resolución de problemas de inclusión jerárquica

\begin{tabular}{|c|c|c|c|c|c|}
\hline $\begin{array}{l}\text { Fuente de } \\
\text { variación }\end{array}$ & $\begin{array}{c}\text { Suma de } \\
\text { cuadrados }\end{array}$ & G. L. & $\begin{array}{c}\text { Media } \\
\text { cuadrática }\end{array}$ & $\mathbf{F}$ & Probabilidad \\
\hline MEAN & 1874.09990 & 1 & 1874.09990 & 6511.23 & 0.0000 \\
\hline CURSO & 42.46265 & 8 & 5.30783 & 18.44 & 0.0000 \\
\hline ERROR & 49.21823 & 171 & 0.28783 & & \\
\hline $\mathrm{R}$ & 28.79617 & 1 & 28.79617 & 211.28 & 0.0000 \\
\hline $\mathrm{RC}$ & 2.50733 & 8 & 0.31342 & 2.30 & 0.0230 \\
\hline ERROR & 23.30634 & 171 & 0.13629 & & \\
\hline$S$ & 0.04049 & 1 & 0.04049 & 0.71 & 0.3996 \\
\hline SC & 0.68901 & 8 & 0.08613 & 1.52 & 0.1545 \\
\hline ERROR & 9.71016 & 171 & 0.05678 & & \\
\hline RS & 0.93657 & 1 & 0.93657 & 14.18 & 0.0002 \\
\hline RSC & 0.64951 & 8 & 0.08119 & 1.23 & 0.2848 \\
\hline ERROR & 11.29675 & 171 & 0.06606 & & \\
\hline $\mathrm{T}$ & 1.23918 & 1 & 1.23918 & 19.08 & 0.0000 \\
\hline TC & 0.99437 & 8 & 0.12430 & 1.91 & 0.0608 \\
\hline ERROR & 11.10787 & 171 & 0.06496 & & \\
\hline RT & 0.81881 & 1 & 0.81881 & 13.59 & 0.0003 \\
\hline RTC & 1.18433 & 8 & 0.14804 & 2.46 & 0.0153 \\
\hline ERROR & 10.30433 & 171 & 0.06026 & & \\
\hline ST & 0.06505 & 1 & 0.06505 & 1.75 & 0.1883 \\
\hline STC & 0.43277 & 8 & 0.05410 & 1.45 & 0.1787 \\
\hline ERROR & 6.37486 & 171 & 0.03728 & & \\
\hline RST & 0.00160 & 1 & 0.00160 & 0.03 & 0.8626 \\
\hline RSTC & 0.96216 & 8 & 0.12027 & 2.26 & 0.0256 \\
\hline ERROR & 9.11053 & 171 & 0.05328 & & \\
\hline \multicolumn{6}{|c|}{$\begin{array}{l}\text { R: Tipo de organización jerárquica. } \\
\text { S: Tipo de presentación. } \\
\text { T: Tipo de categoría. }\end{array}$} \\
\hline
\end{tabular}

TABLA II

Diferencias significativas intergrupo (1)

\begin{tabular}{|c|cccccccccc|}
\hline & \multicolumn{10}{|c|}{ Diferencias } \\
\cline { 2 - 10 } & Medias & Curso & 1 & 2 & 3 & 4 & 5 & 6 & 7 & 8 \\
\hline 0,8645 & 0 & N.S. & N.S. & .200 & .270 & .341 & .412 & .481 & .536 \\
0,9584 & 1 & & N.S. & N.S. & N.S. & .247 & .318 & .387 & .442 \\
0,9969 & 2 & & & N.S. & N.S. & .209 & .279 & .348 & .403 \\
1,0847 & 3 & & & & N.S. & N.S. & .192 & .261 & .316 \\
1.1347 & 4 & & & & & N.S. & N.S. & .211 & .266 \\
1,2058 & 5 & & & & & & N.S. & N.S. & .195 \\
1,2765 & 6 & & & & & & & N.S. & N.S. \\
1,3453 & 7 & & & & & & & & N.S. \\
1,4004 & 8 & & & & &
\end{tabular}

Las diferencias subrayadas son significutivas al 1 por 100 ; las restantes al 5 por 100 .

TABLA III

a) Medias $y$ diferencias significativas intragrupo en clases $(C)$ y colecciones $(K)$

\begin{tabular}{|lcrrrrrrrr|}
\hline Curso & 0 & 1 & 2 & 3 & 4 & 5 & 6 & 7 & 8 \\
\hline $\mathrm{C}$ & .67 & .80 & .81 & .92 & 1.00 & 1.05 & 1.15 & 1.23 & 1.35 \\
$\mathrm{~K}$ & 1.06 & 1.11 & 1.19 & 1.24 & 1.27 & 1.36 & 1.40 & 1.46 & 1.45 \\
\hline Dif. & .39 & .30 & .38 & .32 & .27 & .31 & .25 & .23 & \\
\hline
\end{tabular}


b) Diferencias significativas intergrupo para clases (C) y colecciones $(K)$

\begin{tabular}{|cccccccccc|}
\hline Curso & 0 & 1 & $\mathbf{2}$ & $\mathbf{3}$ & $\mathbf{4}$ & 5 & $\mathbf{6}$ & 7 & $\mathbf{8}$ \\
\hline 0 & & N.S. & N.S. & .257 & .332 & .383 & .484 & .561 & .683 \\
1 & N.S. & & N.S. & N.S. & N.S. & .246 & .348 & .424 & .546 \\
2 & N.S. & N.S. & & N.S. & N.S. & .246 & .347 & .424 & .545 \\
3 & N.S. & N.S. & N.S. & & N.S. & N.S. & .228 & .304 & .426 \\
4 & .207 & N.S. & N.S. & N.S. & & N.S. & N.S. & .229 & .350 \\
5 & .299 & .249 & N.S. & N.S. & N.S. & & N.S. & N.S. & .300 \\
6 & .339 & .288 & .211 & N.S. & N.S. & N.S. & & N.S. & N.S. \\
7 & .400 & .349 & .273 & .217 & N.S. & N.S. & N.S. & & N.S. \\
8 & .389 & .338 & .262 & .206 & N.S. & N.S. & N.S. & N.S. & \\
\hline
\end{tabular}

Las diferencias subrayadas son significativas al l por 100; las no subrayadas, al 5 por 100.

\section{TABLA IV}

Medias correspondientes a los distintos grupos de interacción entre las tres variables estudiadas en cada uno de los nueve niveles escolares

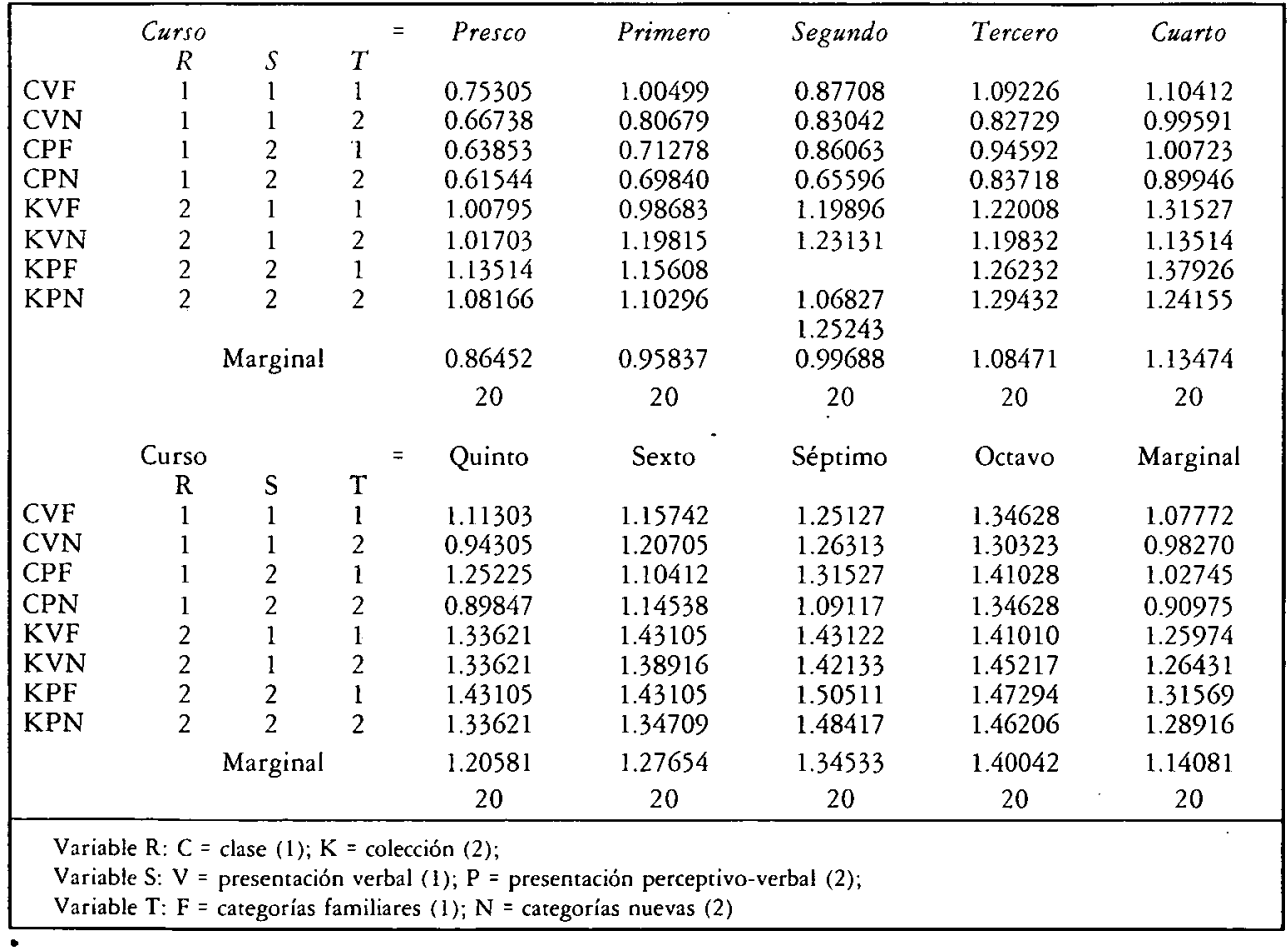

TABLA V

Medias y diferencias significativas intragrupo en $T$ y $A$ para Pertenencia

\begin{tabular}{|lrrrrccccc|}
\hline Curso & \multicolumn{1}{c}{$\mathbf{0}$} & \multicolumn{1}{c}{$\mathbf{1}$} & $\mathbf{2}$ & $\mathbf{3}$ & $\mathbf{4}$ & 5 & $\mathbf{6}$ & $\mathbf{7}$ & $\mathbf{8}$ \\
\hline $\mathrm{T}$ & 2.60 & 2.40 & 2.85 & 1.95 & 2.70 & 2.60 & 2.35 & 3.50 & 2.80 \\
$\mathrm{~A}$ & .55 & .25 & .70 & 1.85 & 1.10 & 1.60 & 1.65 & 2.30 & 2.45 \\
\hline Dif. & 2.05 & 2.15 & 1.15 & & 1.60 & & 1.60 & 1.20 & \\
\hline
\end{tabular}

Los valores subrayados son significarivos al nivel del 1 por 100. 
TABLA VI

Medias $y$ diferencias significativas intragrupo en $T$ y A para Propiedades

\begin{tabular}{|lrrrrrrrrr|}
\hline Curso & 0 & 1 & 2 & 3 & 4 & 5 & 6 & 7 & 8 \\
\hline $\mathrm{T}$ & 1.30 & .95 & 1.30 & 1.05 & 1.15 & 1.45 & 1.60 & 1.80 & 1.25 \\
$\mathrm{~A}$ & .20 & .00 & .35 & .65 & .50 & .65 & .90 & 1.15 & 1.25 \\
\hline Dif. & 1.10 & .95 & .95 & & .65 & .80 & .70 & .65 & \\
\hline
\end{tabular}

Los valores subrayados son significativos al 1 por 100. Los no subrayados, al 5 por 100.

\section{TABLA VII}

Medias $y$ diferencias significativas intragrupo en $F$ y $N$ para Pertenencia

\begin{tabular}{|lccccccccc|}
\hline Curso & 0 & $\mathbf{1}$ & $\mathbf{2}$ & $\mathbf{3}$ & $\mathbf{4}$ & $\mathbf{5}$ & $\mathbf{6}$ & $\mathbf{7}$ & $\mathbf{8}$ \\
\hline $\mathrm{F}$ & 2.10 & 1.55 & 2.20 & 2.15 & 2.10 & 2.30 & 2.50 & 3.15 & 2.95 \\
$\mathrm{~N}$ & 1.05 & 1.10 & 1.35 & 1.65 & 1.70 & 1.90 & 2.40 & 2.65 & 2.30 \\
\hline Dif. & 1.05 & .45 & .85 & & & & & .50 & .65 \\
\hline
\end{tabular}

Las diferencias subrayadas son-significativas al 1 por 100 . Las no subrayadas, al 5 por 100.

\section{TABLA VIII}

Medias $y$ diferencias significativas intragrupo en $F$ y $N$ con cuantificadores

\begin{tabular}{|lrrrrrrrrr|}
\hline Curso & 0 & 1 & 2 & 3 & 4 & 5 & 6 & 7 & 8 \\
\hline $\mathrm{F}$ & 3.05 & 2.95 & 3.20 & 3.10 & 3.55 & 3.50 & 3.85 & 3.65 & 3.70 \\
$\mathrm{~N}$ & 2.15 & 2.15 & 1.85 & 2.25 & 2.00 & 2.35 & 2.50 & 3.15 & 2.80 \\
\hline Dif. & .90 & .80 & .35 & .85 & .55 & .15 & .35 & .50 & .90 \\
\hline
\end{tabular}

Las diferencias subrayadas son significativas al 1 por 100 . Las no subrayadas, al 5 por 100 .

\section{Tabla IX}

Medias transformadas en proporciones para las tres partes de la prueba

\begin{tabular}{|lccccccccc|c|}
\hline Curso & 0 & 1 & 2 & 3 & 4 & 5 & 6 & 7 & 8 \\
\hline $\mathrm{E}$ & .55 & .62 & .65 & .72 & .76 & .80 & .85 & .90 & .93 \\
$\mathrm{C}$ & .38 & .30 & .43 & .46 & .45 & .52 & .61 & .73 & .64 \\
$\mathrm{~T}$ & .65 & .64 & .63 & .64 & .69 & .73 & .79 & .85 & .81 \\
\hline
\end{tabular}

TABla $\mathrm{X}$

Diferencias de proporciones significativas intragrupo en las tres partes $E, C$ y $T$

\begin{tabular}{|lcccccccccc|}
\hline Curso & 0 & 1 & 2 & 3 & 4 & 5 & 6 & 7 & 8 \\
\hline E-T & .16 & .32 & .22 & .26 & .30 & .27 & .23 & .17 & .28 \\
E-C & N.S. & N.S. & N.S. & N.S. & N.S. & N.S. & N.S. & N.S. & .11 \\
T-C & .26 & .34 & .20 & .18 & .24 & .22 & .18 & .12 & .17 \\
\hline
\end{tabular}

Las diferencias se han tomado en valores absolutos.

Los valores subrayados son significativos al 1 por 100 . Los no subrayados, al 5 por 100 . 
GRÁFICA 1

Efecto de la variable curso (C)

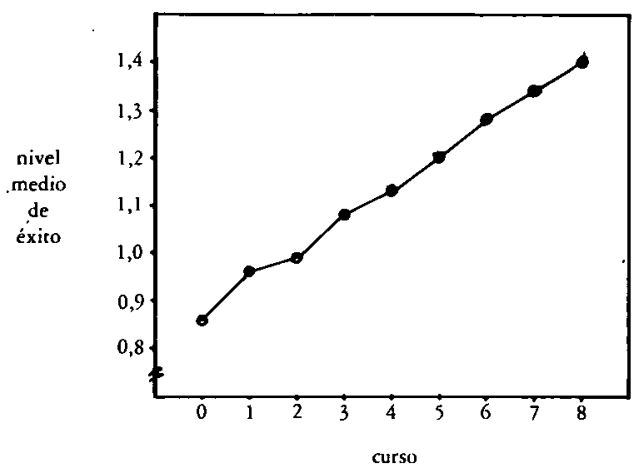

GRÁFICA 2

Efecto de la interacción de las variables tipo de estructura $(R)$ y curso $(C)$

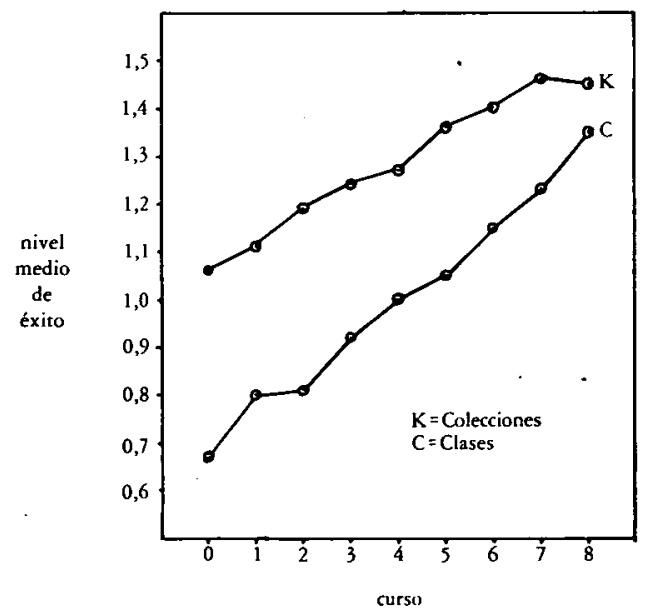

GRÁFICA 3

Efecto de la interacción de las variables tipo de estructura $(R)$ y tipo de presentación (S)

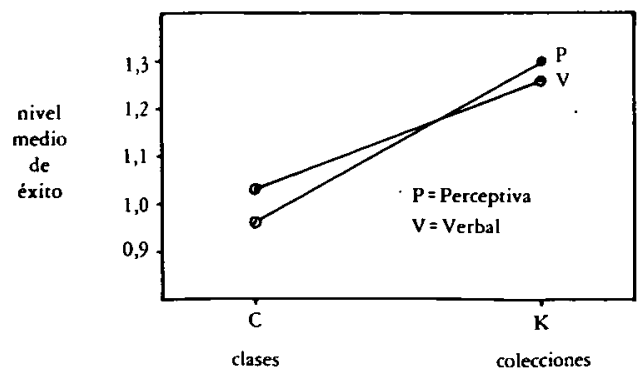

Efecto de la interacción de las variables tipo de estructura $(R)$ y tipo de categoria (T)

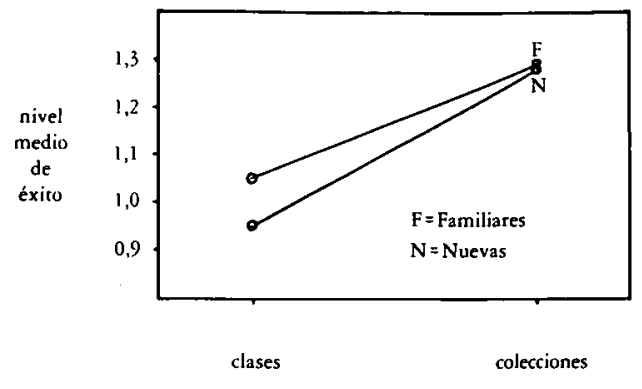

\section{GRÁFICA 5}

Efecto de la interacción de las variables tipo de categoria (T) y curso (C) (no sig.)

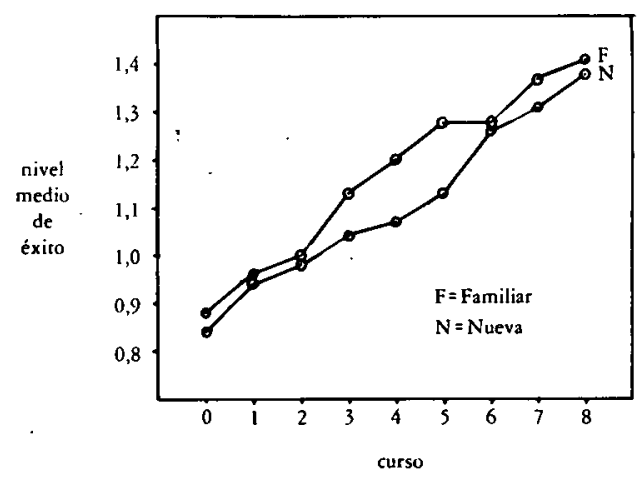

\section{GRÁFICA 6}

Efecto de la variable curso en la ejecución global de Transitividad (T) y parciales de Pertenencia $(P t)$ y Propiedades $(P r)$

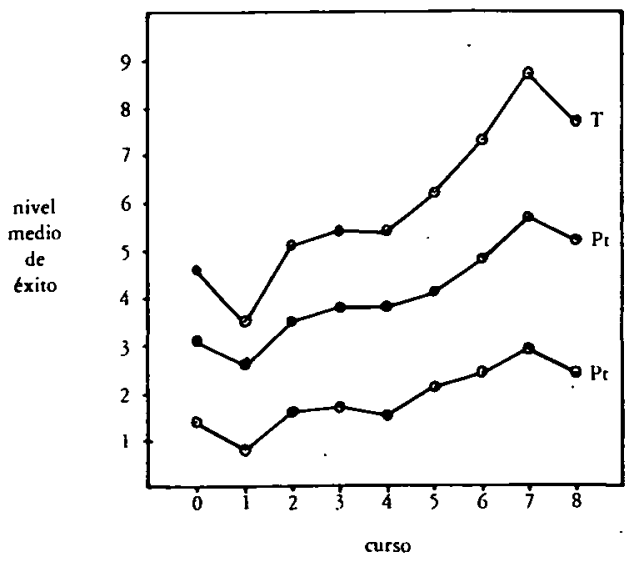


GRÁFICA 7

Ejecución en los cursos según el tipo de cuestión Transitividad directa (T) v.s. Asimetria de la transitividad $(A)$ en cuestiones de Pertenencia

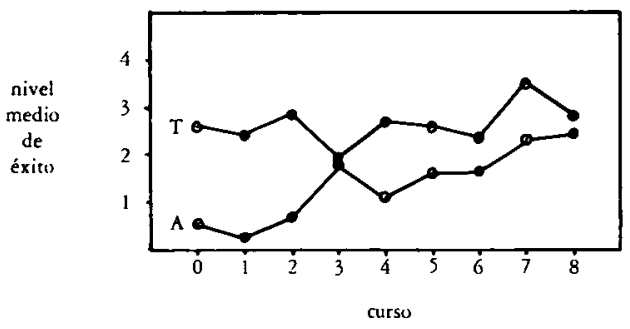

GRÁFICA 8

Ejecución en los cursos en las cuestiones de

Transitividad y Asimetria de Propiedades

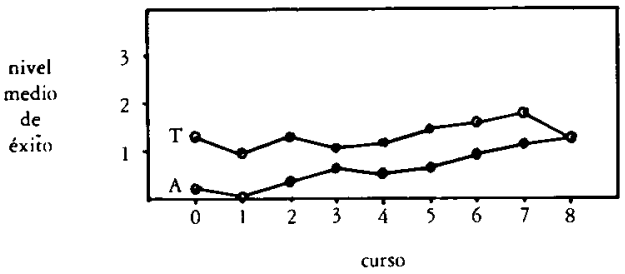

GRÁFICA 9

Ejecución en los cursos según el tipo de formulación: Positiva (+) v.s. Negativa (-) en las cuestiones de Pertenencia

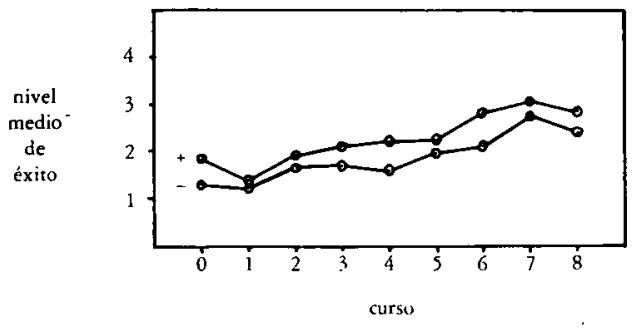

GRÁFICA 10

Ejecución en los cursos según el tipo de categoria: Familiar (F) v.s. Nueva (N) en cuestiones de Pertenencia

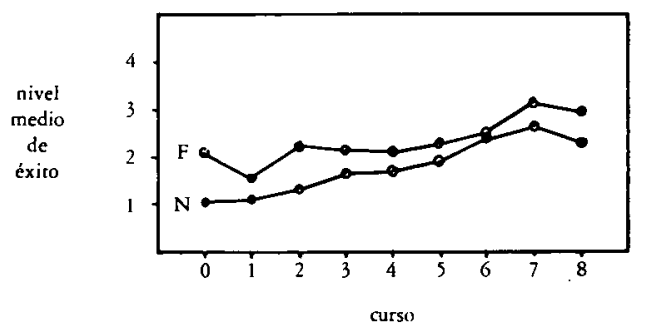

GRÁFICA 11

Ejecución en las cuestiones de cuantificadores según el curso

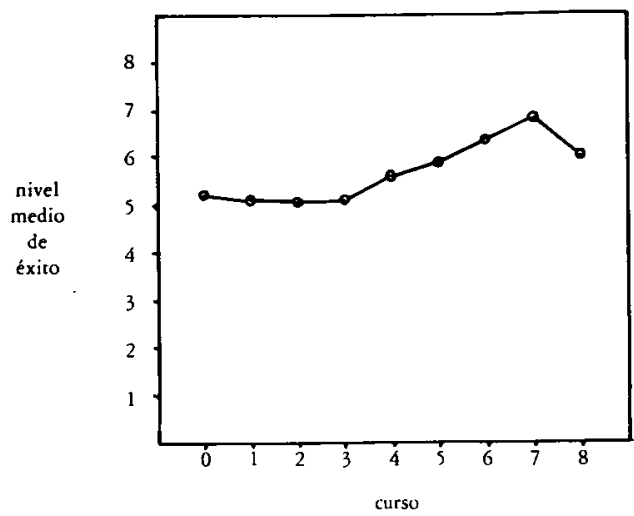

GRÁFICA 12

Ejecución en las cuestiones con cuantificadores según el curso y tipo de categoria: Familiar $(F)$ v.s. Nueva (N)

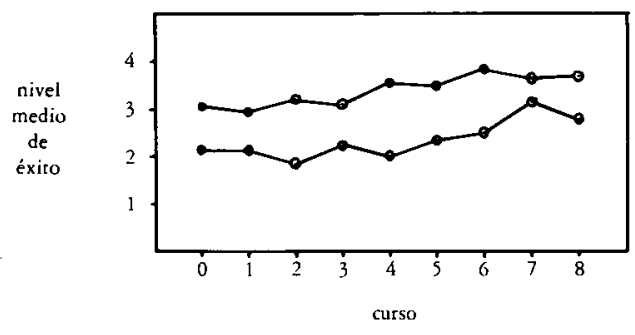

GRÁFICA 13

Ejecución global por cursos en las tres partes de la prueba

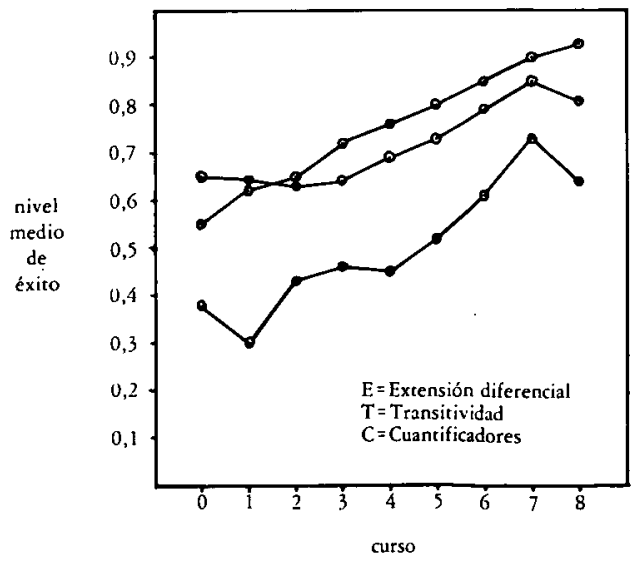




\section{PRUEBA UTILIZADA}

APELLIDOS: NOMBRE:

COLEGIO: CURSO

GRUPO

EDAD:

FECHA DE NACIMIENTO

\section{EN UNA CASA HAY PERROS Y GATOS.}

1 ¿Hay más perros o más animales?

2 ¿Qué quedará si sacamos los perros?

3 ¿Qué quedará si sacamos los animales?

4 Si metemos dos perros más en la casa, ¿habrá más perros o más animales?

5 Si sacamos un gato de la casa, ¿habrá más animales o más perros?
A - más perros.

B - más animales.

A - los gatos.

B - nada.

A - los perros.

B - nada.

C - los gatos.

A - más perros.

B - más animales.

A - más animales.

B - más perros.

EXISTEN UNOS SERES LLAMADOS BALUKAS. HAY BALUKAS DE DOS CLASES LOS BALUKETAS, QUE SON BLANCOS Y LOS BALUMIKOS, QUE SON NEGROS. LOS BALUKETAS SON BALUKAS Y LOS BALUMIKOS SON BALUKAS.

6 ¿Hay más balukas o más baluketas?

7 ¿Qué quedará si se mueren los balukas?

8 ¿Qué quedará si se mueren los baluketas?

9 Si se muere un balumiko, ¿habrá más baluketas o más balukas?

10 Si nacen dos baluketas más, ¿habrá más baluketas o más balukas?
A - más balukas.

B - más baluketas.

A - los baluketas.

B - los balumikos.

C - nada.

A - los balumikos.

B - nada.

A - más balukas.

B - más baluketas.

A - más baluketas.

B - más balukas.

EXISTEN UNOS SERES LLAMADOS MARCILELOS. HAY MARCILELOS DE DOS CLASES: LOS MARCITROMPOS, CON LAS OREJAS EN FORMA DE TROMPETA Y LOS MARCIFANTES, CON LAS OREJAS COMO TROMPAS DE ELEFANTE. LOS MARCITROMPOS SON MARCILELOS Y LOS MARCIFANTES SON MARCILELOS.

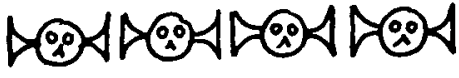

\section{MARCITROMPOS}

11 ¿Hay más marcitrompos o más marcilelos?

12 ¿Qué quedará si se mueren los marcilelos?

13 ¿Qué quedará si se mueren los marcitrompos?

14 Si nacen dos marcitrompos más, ¿habrá más marcilelos o más marcitrompos?

15 Si se muere un marcifante, ¿habrá más marcitrompos o más marcilelos?

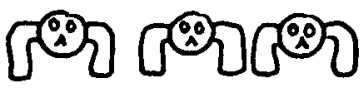

MARCIFANTES

A - más marcitrompos.

B - más marcilelos.

A - los marcifantes.

B - los marcitrompos.

$C$ - nada.

A - nada.

B - los marcifantes.

A - más marcilelos.

B - más marcitrompos.

A - más marcitrompos.

B - más marcilelos. 
EN UN JARDIN HAY TULIPANES Y MARGARITAS.
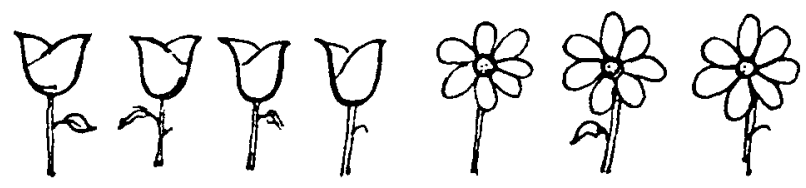

16 ¿Hay más flores o más tulipanes?

A - más tulipanes.

B - más flores.

17 ¿Qué quedará si quitamos los tulipanes?

A - nada.

18 ¿Qué quedará si quitamos las flores?

B - las margaritas.

A - nada.

B - las margaritas.

C - los tulipanes.

19 Si plantamos dos tulipanes más, ¿habrá más flores o más tulipanes?

A - más tulipanes.

B - más flores.

20 Si quitamos una margarita, ¿habrá más flores o más tulipanes?

A - más flores.

B - más tulipanes.

21 Un licaón es un perro; ¿un licaón es un animal?
A - Sí
B - No
C - No se sabe

22 Un raca es un animal; ¿un raca es un gato?
A - Sí
B - No
C - No se sabe

23 Un pilo no es una manzana; ¿un pilo es una fruta?
A - Sí
$\mathrm{B}$ - No
C - No se sabe

24 Un naselo no es una fruta; ¿un naselo es una naranja?
A - S
B - No
C - No se sabe

LOS MARCITROMPOS SON UNA CLASE DE MARCILELOS.

25 Un pepo es un marcitrompo; ¿un pepo es un marcilelo?
A - Sí
B - No
C - No se sabe

26 Un bose es un marcilelo; iun bose es un marcitrompo?
A - Sí B - No
C - No se sabe

\section{LOS TIPALICOS SON UNA CLASE DE TIPACUANOS.}

27 Un nusca no es un tipalico; iun nusca es un tipacuano?

$$
\text { A - Sí B - No C - No se sabe }
$$

28 Un silo no es un tipacuano; ¿un silo es un tipalico?

$$
\text { A - Sí B - No C - No se sabe }
$$

\section{LOS BALUMIKOS SON UNA CLASE DE BALUKAS.}

29 Todos los balukas tienen un ojo; ¿todos los balumikos tienen un ojo?

$$
\text { A - Sí B - No C - No se sabe }
$$

30 Todos los balumikos son altos; ¿todos los balukas son altos?

$$
\text { A - Sí B - No C - No se sabe }
$$

31 Todas las rosas tienen ansore; ¿todas las flores tienen ansore?

$$
\text { A - Sí B - No C - no se sabe }
$$

32 Todas las flores tienen gima; ¿todas las rosas tienen gima?

$$
\text { A - Sí B - No C - No se sabe }
$$

LOS GOLOS SON UNA CLASE DE GOLATES. De acuerdo con esto indica si las siguientes afirmaciones son correctas:

33 Todos los golos son golates.

34 Sólo algunos golos son golates.

35 Sólo algunos golates son golos.

36 Todos los golates son golos.

Sí No

Sí No

Sí No

Sí No

Indica del mismo modo si las siguientes afirmaciones son correctas:

37 Todas las naranjas son frutas.

38 Sólo algunas naranjas son frutas.

39 Sólo algunas frutas son naranjas.

Sí No

40 Todas las frutas son naranjas.

Sí No

Sí No

Sí No


41 ¿Quién tendrá más, el que coja las ovejas o el que coja el rebaño?

42 ¿Qué quedará si nos llevamos las ovejas?

A - el que coja el rebaño.

B - El que coja las ovejas.

43 ¿Qué quedará si nos llevamos el rebaño?

A - las cabras.

B - nada.

A - las ovejas.

B - nada.

C - las cabras.

44 Si nacen dos ovejas más, ¿quién tendrá más, el que coja las ovejas o el que coja el rebaño?

A - el que coja las ovejas.

B - el que coja el rebaño.

45 Si se muere una cabra, ¿quién tendrá más, el que coja el rebaño o el que coja las ovejas?

A - el que coja el rebaño.

$\mathrm{B}$ - el que coja las ovejas.

EXISTEN UNAS COSAS LLAMADAS CELAPOS. HAY CELAPOS DE DOS CLASES: LOS CELAPINOS, QUE SON CORTOS Y LOS CELAPATOS, QUE SON LARGOS. LOS CELAPINOS SON CELAPOS Y LOS CELAPATOS SON CELAPOS. EN UNA MESA TENEMOS CELAPINOS Y CELAPATOS.

46, ¿Quién tendrá más, el que coja el montón o el que coja los celapinos?

47 ¿Qué quedará si cogemos los celapinos?

A - el que coja los celapinos.

B - el que coja el montón.

48 ¿Qué quedará si cogemos el montón?

A - nada.

B - los celapatos.

A - nada.

B - los celapatos.

C - los celapinos.

49 Si quitamos un celapato, ¿quién tendrá más, el que coja los celapinos o el que coja el montón?

A - el que coja los celapinos.

B - el que coja el montón.

$50 \mathrm{Si}$ ponemos dos celapinos más, ¿quién tendrá más, el que coja los celapinos o el que coja el montón?

A - el que coja el montón.

B - el que coja los celapinos.

EN UNA MESA TENEMOS PELOTAS PEQUEÑAS Y GRANDES.

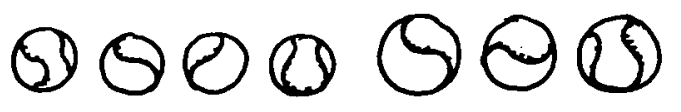

51 ¿Quién tendrá más, el que coja las pelotas pequeñas o el que coja el montón?

52 ¿Qué quedará si quitamos el montón?

53 ¿Qué quedará si quitamos las pelotas pequeñas?

54 Si ponemos dos pelotas grandes más, ¿quién tendrá más, el que coja las pelotas pequeñas o el que coja el montón?

55 Si quitamos una pelota grande, ¿quién tendrá más, el que coja las pelotas pequeñas o el que coja el montón?

A - el que coja el montón.

$B$ - El que coja las pelotas pequeñas.

A - nada.

B - las pelotas pequeñas.

C - las pelotas grandes.

A - nada.

B - las pelotas grandes.

A - el que coja el montón.

B - el que coja las pelotas pequeñas.

A - el que coja las pelotas pequeñas. $B$ - el que coja el montón.

EXISTEN UNAS COSAS LLAMADAS TIPACUANOS. HAY TIPACUANOS DE DOS CLASES: LOS TIPACONOS QUE SON NEGROS Y LOS TIPALICOS QUE SON BLANCOS. LOS TIPACONOS SON TIPACUANOS Y LOS TIPALICOS SON TIPACUANOS. EN UNA MESA TENEMOS TIPACONOS Y TIPALICOS.

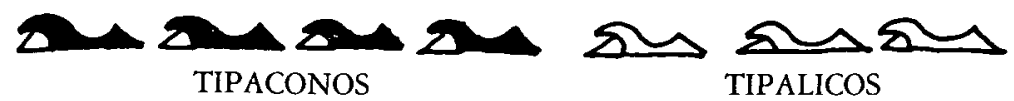

56 ¿Quién tendrá más, el que coja el montón o el que coja los tipaconos?

¿Qué quedará si quitamos los tipaconos?

A - el que coja los tipaconos.

B - el que coja el montón.

A - los tipalicos.

B - nada. 
58 ¿Qué quedará si quitamos el montón?

$$
\begin{aligned}
& \text { A - los tipalicos. } \\
& \text { B - los tipaconos. } \\
& \text { C - nada. }
\end{aligned}
$$

59 Si quitamos un tipalico, ¿quién tendrá más, el que coja el montón o el que coja los tipaconos?

A - el que coja los tipaconos.

B - el que coja el montón.

60 Si ponemos dos tipaconos más, ¿quién tendrá más, el que coja el montón o el que coja los tipaconos?

A - el que coja los tipaconos.

B - el que coja el montón.

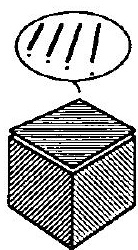

\section{Anexo II}

\section{HOJA DE RESPUESTAS}

APELLIDOS:

NOMBRE:

COLEGIO:

CURSO

GRUPO:

FECHA DE NACIMIENTO:

EDAD:

\begin{tabular}{rrrlllll}
\hline 1. & A & B & C & 31. & A & B & C \\
2. & A & B & & 32. & A & B & C \\
3. & A & B & C & 33. & SI & NO & \\
4. & A & B & & 34. & SI & NO & \\
5. & A & B & & 35. & SI & NO & \\
6. & A & B & & 36. & SI & NO & \\
7. & A & B & C & 37. & SI & NO & \\
8. & A & B & C & 38. & SI & NO & \\
9. & A & B & & 39. & SI & NO & \\
10. & A & B & & 40. & SI & NO & \\
11. & A & B & & 41. & A & B & \\
12. & A & B & C & 42. & A & B & \\
13. & A & B & & 43. & A & B & C \\
14. & A & B & & 44. & A & B & \\
15. & A & B & & 45. & A & B & \\
16. & A & B & & 46. & A & B & \\
17. & A & B & & 47. & A & B & C \\
18. & A & B & C & 48. & A & B & \\
19. & A & B & & 49. & A & B & \\
20. & A & B & & 50. & A & B & \\
21. & A & B & C & 51. & A & B & C \\
22. & A & B & C & 52. & A & B & \\
23. & A & B & C & 53. & A & B & \\
24. & A & B & C & 54. & A & B & \\
25. & A & B & C & 55. & A & B & \\
26. & A & B & C & 56. & A & B & \\
27. & A & B & C & 57. & A & B & C \\
28. & A & B & C & 58. & A & B & \\
29. & A & B & C & 59. & A & B & \\
30. & A & B & C & 60. & A & B & \\
\hline
\end{tabular}

Anexo III

\section{INSTRUCCIONES PARA LA PRUEBA}

En los cuadernillos que os he repartido, hay una serie de ejercicios en los cuales debéis responder unas preguntas.

Cada ejercicio es diferente de los demás —aunque en muchos de ellos las preguntas son semejantes- y está separado del siguiente por dos líneas juntas. 
La mayoría tienen al comienzo una presentación escrita en letras mayúsculas y a veces acompañada de dibujos. Las preguntas subsiguientes se refieren, en todos los casos, al contenido expresado en esta presentación inicial.

A continuación de cada pregunta vienen expresadas las alternativas o posibilidades de respuesta, precedidas de una letra A, B o C. De ellas deberéis elegir la que consideréis correcta y la indicaréis en la hoja de respuestas que os he dado aparte, haciendo un círculo alrededor de la letra correspondiente a vuestra elección.

Las preguntas están numeradas para que en la hoja contestéis en su número y no en otro. Si en algún caso os equivocáis de letra al hacer el círculo, la forma de rectificar y corregirlo será hacer una cruz en la equivocada y redondear de nuevo en la letra que creéis correcta.

Debéis tener siempre en cuenta que cada pregunta es independiente de las demás y que se refiere exclusivamente a lo escrito en letras mayúsculas al principio. Así pues, responded cada pregunta por separado, olvidándoos de las anteriores.

En muchos de los ejercicios encontraréis palabras raras. Simplemente son nombres que hemos puesto a seres o cosas imaginarias que hemos inventado. Lo que tenéis que saber de ellas está escrito y debéis responder conforme a lo que se expresa.

Mientras resolvéis el ejercicio podéis preguntarme si encontráis alguna dificultad. ¿Queréis hacerme alguna pregunta antes de comenzar?

\section{Notas}

1 En la introducción teórica de cada experimento justificamos brevemente la elección de estos factores, ver no obstante Markman y Callanan (1984), Alonso y Gutiérrez (1986) para una mayor fundamentación teórica de estas importantes variables.

${ }^{2}$ Las otras dos formas de control propuestas por Markman (1978) quedan incorporadas a las condiciones de presentación verbal (se eliminan los indicios empíricos de número) y de categorías nuevas (no existe conocimiento empírico).

\section{Resumen}

En el presente trabajo se estudia el desarrollo de la comprensión de los principios que rigen la inclusión jerárquica de clases en sujetos de 4 a 14 años. Se presentan y comparan los resultados de tres experimentos en los que se utilizan, respectivamente, cuestiones de tipo piagetiano, problemas deductivos y problemas de inclusión con los cuantificadores "todos" $y$ "algunos". Se examina también el efecto que diferentes variables definitorias de la estructura de las tareas tienen en el éxito o el fracaso en las mismas. Los resultados sugieren un desarrollo lineal progresivo en relación con la comprensión de la extensión diferente de clase y subclase, y la aplicación correcta de los cuantificadores, y un salto entre los 9 y 12 años en relación con la comprensión de la transitividad. Se discuten las implicaciones teóricas y prácticas de estos resultados.

\section{Summary}

In this paper, development of class inclusion comprehension in subjects between four and fourteen years of age is studied. Results from three experiments with different kinds of inclusion problems - piagetian ques. tions, deductive problems and problems with the quantificators "some and all"- are reported and compared. The effect of different variables defining task structure is examined, too. Results suggest a progressive regular lineal development of differential extension between class and subclass comprebension and of correct applica. tiono of quantificators, and a jump between nine and twelve years of age in relation to transitivity comprehension. Theoretical and practical implications are discussed.

\section{Résumé}

Dans cet article on étude le développement de la comprébension des principes logiques de l'inclusion bierarchique de classes avec des sujets dont l'âge est compris entre 4 et 14 ans. On présente et compare les résul. tats de trois études dans lesquels on utilise, respectivement, variations des problémes idées par Piaget, problèmes déductifs et problèmes avec des quantificateurs "quelques" et "tous". On examine aussi l'influence des différentes variables qui definissent la structure des taches on le reussite et l'échec obtenue par des sujets. Les résultats sugérent que le développement de la comprehension des différences d'extension entre classe et subclasse et du correct utilisation des quantificateurs et linéaire et progressif, tandis que la comprébension de la transitivité augmente et se généralise dépuis neuf jusqu'à douze ans. On comment les implications théoretiques et pratiques de ces résultats. 
Alonso Tapia, J. y Gutiérrez Martinez, F.: «Comprensión de la inclusión jerárquica de clases», en J. Alonso (Dir.): «Entrenamiento de habilidades cognitivas y enriquecimiento motivacional: nuevas tecnologías para la educación compensatoria». Memoria final de la primera fase, vol. II, Madrid, C.I.D.E., 1986.

Bruner, J. S.; Olver, R., y Greenfield,« P.: «Studies in cognitive growth». Nueva York: Wiley, 1966.

Evans, J.: "The psychology of deductive reasoning». Londres: Roudledge and Kagan Paul, 1982.

GARDNER, W.R.: The processing of information and structure", Potomac, Md.: Lawrence Erlbaum Associates, 1974.

GARDNER, W. R.: «Aspects of a stimulus: features, dimensions and configurations», en Rosch y LLoID, (Eds.): Cognition and categorization. Hillsdale, N. I.: Lawrence Erlbaum Associates, 1978.

Gelman, R. y Baillargeon, R.: «A review of some piagetian concepts», en Mussen (ed.): Handbook of Child Psychology, vol. 3, cap. 3, Nueva York: Wiley, 1983.

HARris, P. L. y BASSETt, E.: «Transitive inferences by-4-year-old children?», Developmental Psychology, 1975, 11, 875-876.

INHEIDER, B. y PIAGET, J.: The early growth of logic in the child, Nueva York: W. W. Norton, 1964.

INHELDER, B. y PIAGET, J.: The early growth of logic in the child: classification and seriation. Nueva York: Norton, 1964 .

KEIL, F. C.: Semantic and conceptual development: an ontological perspective. Cambridge, Mass.: Harvard University Press, 1979.

MARKman, E. M.: «Facilitation of part-whole comparisons by use of the collective noun family, Child Development, 1973, 44, 837-840.

MARKMAN, E. M. y SELBERT, J.: «Classes and collections: internal organization and resulting holistic properties», Cognitive Psychology, 1976, 8, 561-577.

MARKMAN, E. M. «Empirical versus logical solutions to part-whole comparison problems concerning classes and collections", Child development, 1978, 49, 168-177.

Markman, E. M.; Horton, M. S. y MCLANahan, A. G.: «Classes and collections: principles of organization in the learning of hierachical relations», Cognition, 1980, 8, 227-241.

Markman, E. M.; Cox, B., y MaCHIDA, S.: "The standar object-sorting task as a measure of conceptual organization», Developmental Psychology, 1981, 17, 115-117.

Markman, E. M., y CaLlanan, M. A.: «An analysis of hierarchical classification», en J. Sternberg: Advances in the psychology of buman intelligence, vol. 2, Londres: LEA, 1984.

Oerter, R.: Psicología del pensamiento, Barcelona: Herder, 1975.

Rosch, E.: Mervis, C. B.; Gay, W. D.; Boyes-Braem, P., y Johnson, D. N.: «Basic objects in natural categories», Cognitive Psychology, 1976, 8, 382-439.

Rosch, E. H.: «Principles of categorization», en E. H. Rosch y B. B. Lloyd (eds.): Cognition and categorization», Hillsdale, N. J.: Lawrence Erlbaum Associates, 1978.

Savage-Rumbaugh, E. S.; Rumbaugh, D. M.; Smith, S. T. y Lawson, J.: Reference: the linguistic essential.

SMITH, C. L.: «Children's understanding of natural language hierarchies», Journal of Experimental Child Psycho$\log y, 1979,27,437-458$

SMith, E.E. y MEDin, D. L.: Categories and concepts. Cambridge, Mass.: Harvard University Press, 1981.

VEGA, M.: Introducción a la psicología cognitiva. Madrid: Alianza, 1984.

WINER, G. A.: «Class inclusion reasoning in children: a review of empirical literature», Child Development, $1980,51,309-328$. 